\title{
LKB1 loss in melanoma disrupts directional migration toward extracellular matrix cues
}

\author{
Keefe T. Chan, ${ }^{1,2,7}$ Sreeja B. Asokan, 1,2,7 Samantha J. King, ${ }^{1,2}$ Tao Bo, 1,7 Evan S. Dubose, ${ }^{1,2,7}$ Wenjin Liu, ${ }^{1,3}$ \\ Matthew E. Berginski, ${ }^{4}$ Jeremy M. Simon, ${ }^{1,2,3,5}$ lan J. Davis, ${ }^{1,3,6}$ Shawn M. Gomez, ${ }^{4}$ Norman E. Sharpless, ${ }^{1,3}$ \\ and James E. Bear ${ }^{1,2,7}$
}

'University of North Carolina Lineberger Comprehensive Cancer Center, ${ }^{2}$ Department of Cell Biology and Physiology, ${ }^{3}$ Department of Genetics, ${ }^{4}$ Department of Biomedical Engineering, ${ }^{5}$ Carolina Center for Genome Science, ${ }^{6}$ Department of Pediatrics, and ${ }^{7}$ Howard Hughes Medical Institute, University of North Carolina-Chapel Hill, Chapel Hill, NC 27599

$\mathrm{S}$ omatic inactivation of the serine/threonine kinase gene $S T K 11 / L K B 1 / P A R-4$ occurs in a variety of cancers, including $\sim 10 \%$ of melanoma. However, how the loss of LKB1 activity facilitates melanoma invasion and metastasis remains poorly understood. In LKB Inull cells derived from an autochthonous murine model of melanoma with activated Kras and $L k b l$ loss and matched reconstituted controls, we have investigated the mechanism by which LKB1 loss increases melanoma invasive motility. Using a microfluidic gradient chamber system and time-lapse microscopy, in this paper, we uncover a new

\section{Introduction}

Germline mutations in STK11/LKB1 (Hemminki et al., 1998) are associated with Peutz-Jeghers syndrome (Jeghers et al., 1949), an autosomal-dominant disease characterized by gastrointestinal hamartomatous polyps and hyperpigmentation of the oral mucosa. Patients with Peutz-Jeghers syndrome have enhanced susceptibility to many cancers (Olschwang et al., 2001; Lim et al., 2003; Hearle et al., 2006). Somatic mutations that result in the inactivation of $L K B 1$ are also found in sporadic cancers such as lung adenocarcinoma (Sanchez-Cespedes et al., 2002; Ji et al., 2007), cervical carcinoma (Wingo et al., 2009), pancreatic cancer (Su et al., 1999), and melanoma (Guldberg et al., 1999; Rowan et al., 1999).

Several lines of evidence support a critical role of LKB1 as a tumor suppressor (Sanchez-Cespedes et al., 2002; McCarthy et al., 2009; Miyoshi et al., 2009), but work in murine models, in particular, has shown a prominent role of LKB1 in suppressing

Correspondence to James E. Bear: jbear@email.unc.edu

Abbreviations used in this paper: 4-OHT, 4-hydroxy-tamoxifen; AMPK, AMPactivated protein kinase; ANOVA, analysis of variance; BRSK, brain-specific kinase; DN, dominant negative; FMI, forward migration index; MARK, microtubule affinity-regulating kinase; MMP, matrix metalloproteinase; NUAK, nua kinase; PDMS, polymethylsiloxane; qRT-PCR, quantitative RT-PCR; SIK, salt-inducible kinase; YAP, Yes-associated protein. function for $L K B 1$ as a directional migration sensor of gradients of extracellular matrix (haptotaxis) but not soluble growth factor cues (chemotaxis). Systematic perturbation of known LKB1 effectors demonstrated that this response does not require canonical adenosine monophosphateactivated protein kinase (AMPK) activity but instead requires the activity of the AMPK-related microtubule affinity-regulating kinase (MARK)/PAR-1 family kinases. Inhibition of the LKB1-MARK pathway facilitated invasive motility, suggesting that loss of the ability to sense inhibitory matrix cues may promote melanoma invasion.

metastasis. For example, Kras expression rapidly cooperates with inactivation of several other tumor suppressor genes (e.g., p53 and p16/INK4a) to promote locally aggressive melanoma or nonsmall cell lung cancer, but only $L k b 1$-deficient tumors exhibit widespread hematogenous and lymphatic metastasis (Ji et al., 2007; Carretero et al., 2010; Liu et al., 2012). In both models, $L k b 1$ inactivation is associated with increased expression of CD24, expansion of tumor-initiating fractions, and activation of Src family kinases, but the direct mechanism whereby LKB1 loss facilitates metastasis is poorly understood. This increased propensity of $L k b l$-deficient tumors for advanced local growth and early metastatic spread has been supported by recent human studies (Wingo et al., 2009; Kline et al., 2011; Liu et al., 2013).

LKB1 is a highly conserved serine/threonine kinase that phosphorylates a consensus motif found in 14 different kinases (Lizcano et al., 2004). LKB1 function is most intimately tied to AMP-activated protein kinase (AMPK), a central regulator of

(C) 2014 Chan et al. This article is distributed under the terms of an Aftribution-NoncommercialShare Alike-No Mirror Sites license for the first six months after the publication date (see http://www.rupress.org/terms). After six months it is available under a Creative Commons License (Attribution-Noncommercial-Share Alike 3.0 Unported license, as described at http://creativecommons.org/licenses/by-nc-sa/3.0/1. 
cellular metabolism under conditions of energy stress (Shaw et al., 2004b). However, it is becoming increasingly clear that LKB1 has many AMPK-independent functions in morphogenesis (Rodríguez-Fraticelli et al., 2012) and cancer (Lo et al., 2012; Nguyen et al., 2013). In addition to AMPK, LKB1 activates the brain-specific kinases (BRSKs; BRSK1 and BRSK2), microtubule affinity-regulating kinases (MARKs; MARK1, MARK2, MARK3, and MARK4), salt-inducible kinases (SIKs; SIK1, SIK2, and SIK3), and nua (novel)/SNF1-like kinases (NUAK1 and NUAK2), which participate in multiple cellular functions, such as anoikis, actomyosin contractility, and cell polarity (Marcus and Zhou, 2010). For instance, in neurons, LKB1 not only promotes axon specification through BRSKs (Barnes et al., 2007; Shelly et al., 2007) but also controls axon branching via NUAK1 (Courchet et al., 2013).

Genetic studies in Caenorhabditis elegans (Watts et al., 2000) and Drosophila melanogaster (Martin and St Johnston, 2003) first identified a key role of LKB1 in the establishment of cell polarity, which has since been extended to mammalian systems (Baas et al., 2004). This is most evident in epithelial cells in which LKB 1 activity is required to maintain apical-basal polarity in the intestine (Baas et al., 2004), pancreas (Hezel et al., 2008), and mammary gland (Partanen et al., 2012). Loss of apical-basal polarity is thought to be a quintessential characteristic of epithelial-derived cancer, which occurs during epithelial-mesenchymal transition (Chaffer and Weinberg, 2011). However, murine tumor models with LKB1 loss show loss of apical-basal polarity in some but not all (Contreras et al., 2008; Lo et al., 2012) cancers, suggesting that LKB1 has contextdependent functions.

LKB1 is also found in more motile mesenchymal cells, which typically display a front-rear polarity that spontaneously allows cells to migrate (Ridley et al., 2003). Cells must establish this asymmetry during directed migration toward soluble growth factor (chemotaxis), surface-bound ECM (haptotaxis), and mechanical cues (durotaxis; Petrie et al., 2009). Guiding principles have emerged to describe how directional migration is orchestrated, which include actin polymerization, stabilization of adhesions, focalized proteolysis, cell contractility, and detachment (Friedl and Alexander, 2011). Furthermore, significant technological advances have enabled more rigorous investigation of directional cell migration (Shamloo et al., 2008; Wu et al., 2012). Despite recent progress, how LKB1 participates in regulating directional cell migration remains incompletely understood. Based on the finding that loss of LKB1 promotes metastasis in several tumor types, here, we seek to interrogate the cell biological basis by which LKB1 controls migration and invasion in melanoma.

\section{Results}

Loss of LKB1 does not affect invadopodia formation in melanoma cells

Given the potent effect of LKB1 loss on invasion and metastasis, we expected that LKB1 loss would promote the formation of invadopodia, the matrix-degrading organelles often formed by metastatic cancer cells (Chen, 1989; Yamaguchi et al., 2005).
To investigate this, we depleted LKB1 in the human melanoma cell line A2058 (BRAF ${ }^{V 600 E} / P T E N$ null/RB1 null) and the mu-

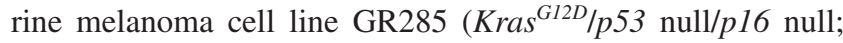
Fig. 1 A; Monahan et al., 2010) and tested their ability to form invadopodia on the fluorescent gelatin matrix (Fig. 1 B). Surprisingly, we found no difference in the percentage of cells forming invadopodia in either melanoma cell line with LKB1 depletion (Fig. 1 C).

We considered the possibility that subtle differences might be missed in this fixed time point assay. To test this more rigorously, we performed live-cell time-lapse microscopy of the wellcharacterized human melanoma cell line WM-266-4 expressing an actin marker Lifeact-GFP plated on Alexa Fluor 568-gelatin over a period of $27 \mathrm{~h}$ (Video 1). We then developed an unbiased automated vision approach to classify "degraders" and "nondegraders" from multiple imaging fields (Berginski et al., 2014). As expected, the percentage of cells with invadopodia increased as a function of time (Fig. 1 D). However, similar to our results obtained from fixed time points, we observed no significant differences in the percentage of cells forming invadopodia with or without LKB1.

As an additional approach, we used lentiviral/retroviral reconstitution of $\mathrm{Kras}^{G 12 D} / \mathrm{Lkbl}$-null melanoma cells established from a recently described autochthonous murine model of metastatic melanoma (Fig. S1 A; Liu et al., 2012). We confirmed that reexpression of LKB1 (addback) promoted phosphorylation of AMPK and its downstream effector acetyl-CoA carboxylase in three LKB1-deficient cell lines (LKB498, LKB878, and TKL2; Fig. S1 B). We co-cultured these LKB1-null and reconstituted melanoma cells with WM-266-4 cells (as a positive control) on fluorescent gelatin (Fig. 1 E). We observed robust matrix-degrading invadopodia in WM-266-4 cells but surprisingly found no invadopodia in LKB498-null or addback cells or in the two other LKB1-deficient melanoma cell lines (LKB878 and TKL2) derived from the same genetic model (unpublished data). Together, these data indicate that the enhanced invasion and metastasis resulting from the loss of LKB1 likely do not rely on differences in invadopodia formation but instead reflect a different property of the cells.

\section{LKB1 limits single-cell and} collective migration

The finding that LKB1 does not appear to influence the proportion of cells forming invadopodia prompted us to more closely examine its effects on cell migration. We first assessed random single-cell motility in 3D collagen and found that LKB1 addback reduced cell speed by a modest $30 \%$ (Fig. 2 A). To test the effect of LKB1 addback on 3D migration in a collective context, we generated tumor cell spheroids and examined invasion into 3D collagen gels (Fig. 2 B). At all concentrations tested, we found reduced invasive outgrowth in cells with LKB1 addback and a further reduction as compared with null cells at higher collagen concentrations (Fig. 2 C). We next investigated motility on 2D surfaces. One prominent characteristic of mesenchymal cells is their ability to migrate on ECM substratum in a biphasic fashion, with optimal cell speed at intermediate matrix concentrations (Goodman et al., 1989; DiMilla et al., 1993). 


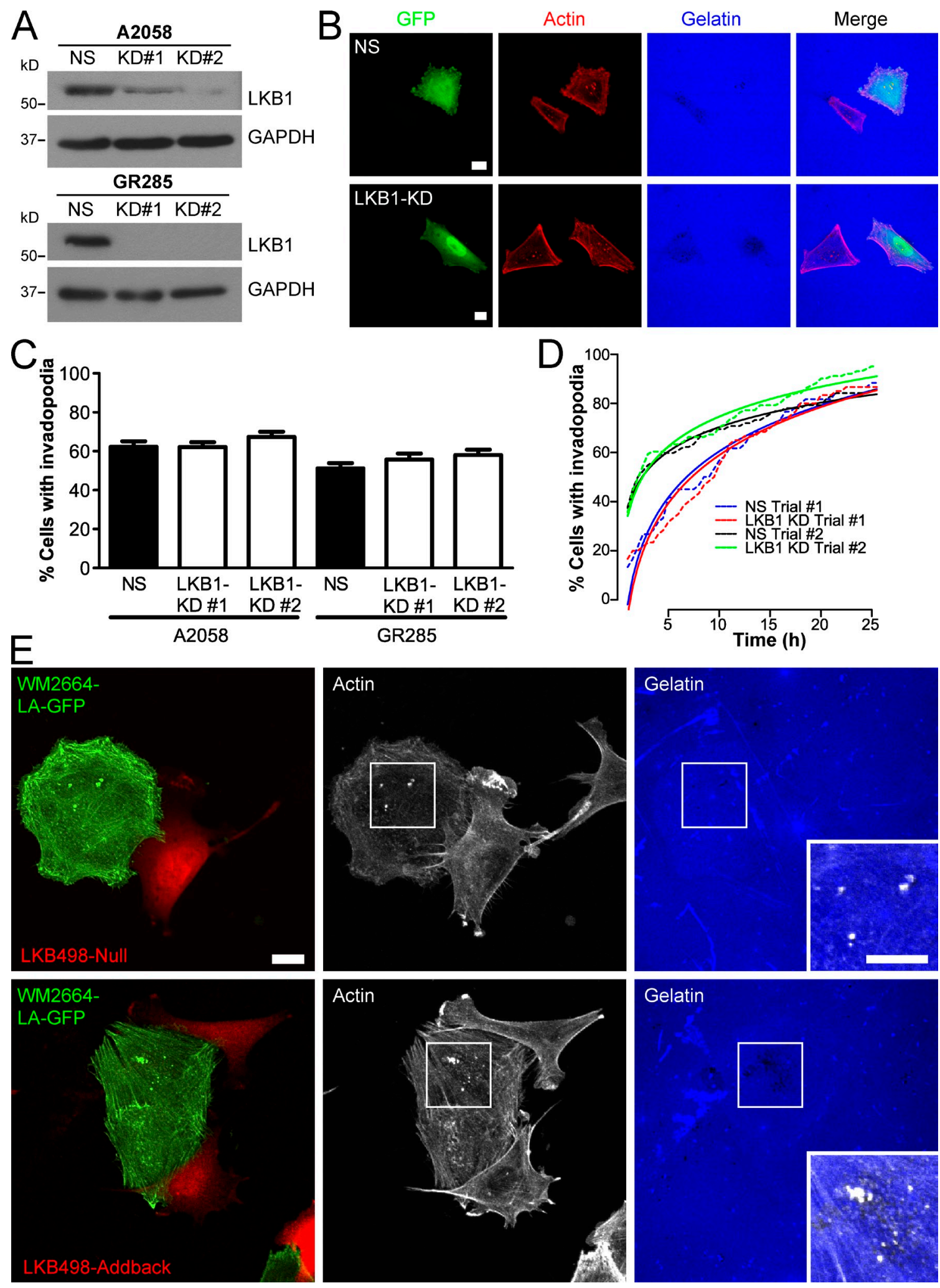

Figure 1. Loss of LKB1 does not affect invadopodia formation in melanoma cells. (A) Western blot showing lentiviral shRNA knockdown of a nontargeting sequence (NS) or LKB1 (knockdown [KD]) in human A2058 (BRAFV600E/RB null) and mouse GR285 (Kras ${ }^{G 12 D} / \mathrm{p} 53$ null/p16 null) melanoma. (B) Invadopodia formation on Alexa Fluor 647-gelatin. GFP is a marker of knockdown, and cells were stained with Alexa Fluor 568-phalloidin to label actin. (C) Percentage of cells with invadopodia shown as means \pm SEM. (A2058: nontargeting sequence, $n=297 ; \mathrm{KD} \# 1, n=413$; KD\#2, $n=300$. GR285: nontargeting sequence, $n=321 ; K D \# 1, n=255 ; K D \# 2, n=312$.) (D) Live-cell invadopodia assay shows no difference in invadopodia formation over time. Dashed lines show raw data and solid lines are best-fit curves to the data. (Trial \#1: nontargeting sequence, $n=60 ;$ LKB1 knockdown, $n=60$. Trial \#2: nontargeting sequence, $n=82$, LKB1 knockdown, $n=101$.) (E) Invadopodia assay on Alexa Fluor 405-gelatin of WM-266-4 (BRAFV600D/PTEN null) Lifeact (LA)-GFP cells and tdTomato-labeled LKB498 (Kras G12D/Lkb 1 null) or LKB1 addback melanoma. Cells were stained with Alexa Fluor 647-phalloidin to label actin. Insets show magnified region of invadopodia. Bars, $20 \mu \mathrm{m}$. 

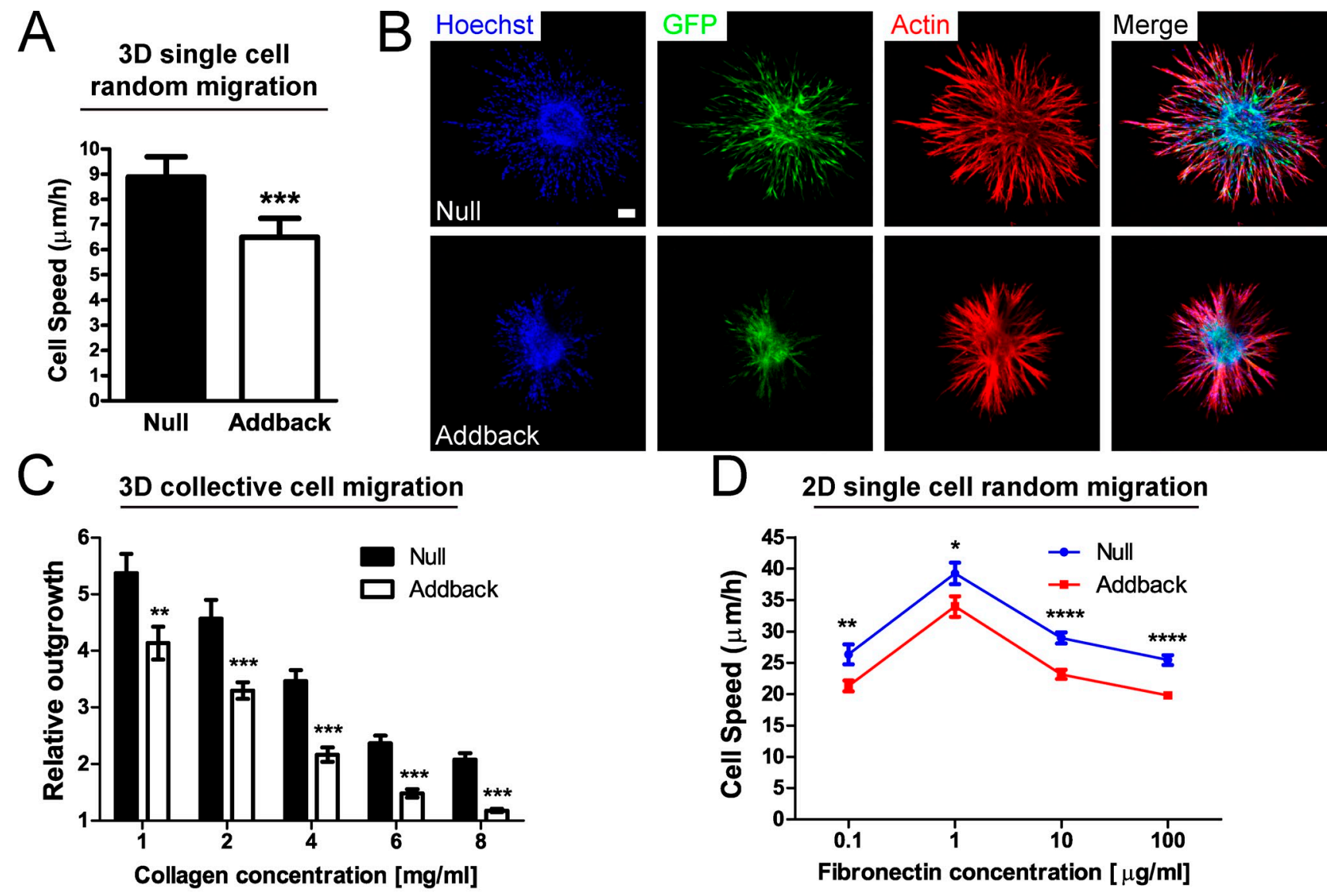

\section{D collective cell migration}
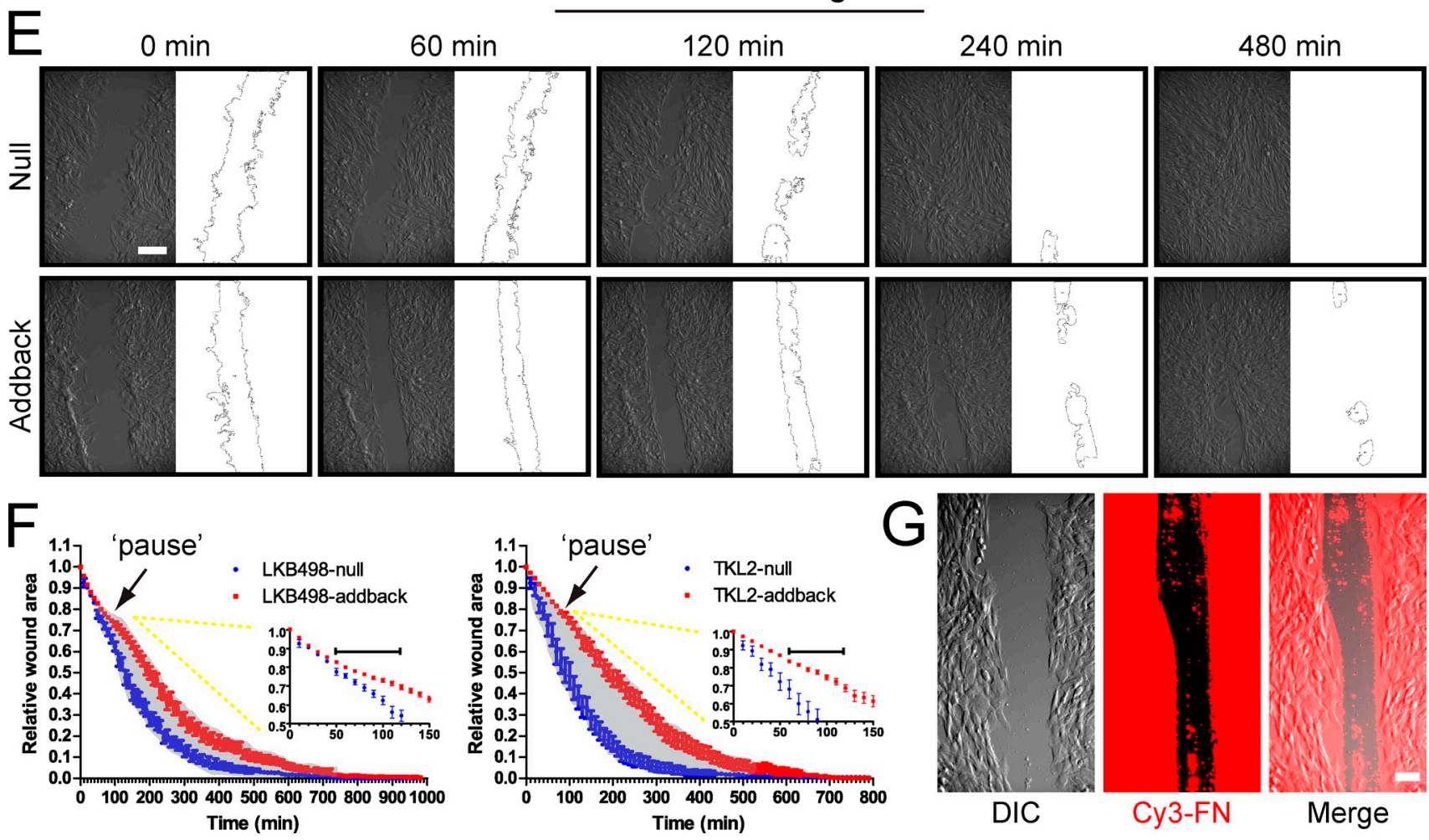

Figure 2. LKB1 limits single-cell and collective cell migration in melanoma. (A) Single-cell speed of LKB498 cells in $1 \mathrm{mg} / \mathrm{ml} 3 \mathrm{D}$ collagen. (null, $n=73$; addback, $n=83$.) (B) Spheroid invasion of LKB498 cells into $3.2 \mathrm{mg} / \mathrm{ml} 3 \mathrm{D}$ collagen. Cells were stained with Alexa Fluor 568-phalloidin to label actin and Hoechst 33342 to label nuclei. (C) Spheroid outgrowth of LKB498 cells into increasing concentrations of 3D collagen. (1 mg/ml: null, $n=22 ;$ addback, $n=24.2 \mathrm{mg} / \mathrm{ml}$ : null, $n=21$; addback, $n=27.4 \mathrm{mg} / \mathrm{ml}:$ null, $n=30$; addback, $n=29.6 \mathrm{mg} / \mathrm{ml}: \mathrm{null}, n=20 ;$ addback, $n=23.8 \mathrm{mg} / \mathrm{ml}$ : 
At all matrix concentrations tested, LKB1 addback reduced single-cell migration speed (Fig. 2 D). However, regardless of LKB1 status, cells displayed biphasic motility with optimal migration occurring on $1 \mu \mathrm{g} / \mathrm{ml}$ fibronectin. This suggests that, despite the reduction in cell speed observed upon reintroduction of LKB1, cells with or without LKB1 can still respond similarly to absolute differences in ECM concentration.

To assay collective cell migration in $2 \mathrm{D}$, we performed scratch wound assays of cell monolayers, and by time-lapse microscopy, we used a method to automatically detect the collective cell margin and quantify wound closure over time. Consistent with previous work (Carretero et al., 2010; Liu et al., 2012), we observed delayed closure upon reintroduction of LKB1 compared with the null cells (Fig. 2 E). Upon careful examination of the time-lapse videos (Video 2 and Video 3), we noticed that cells with LKB1 addback paused for over an hour and lined up parallel to the wound edge (Fig. 2 F). In contrast, LKB1-null cells completely ignored this boundary and migrated directly into the cleared area without delay. During the generation of a scratch wound, the underlying ECM is often removed, and we speculated that LKB1 addback cells were aligning with this discontinuous ECM. To test this notion, we performed a scratch wound on an LKB1 addback monolayer plated on fluorescent fibronectin and observed a strong tendency for LKB1 addback cells to align with the ECM boundary (Fig. 2 G). These data highlight the ability of LKB1 to limit cell migration both in single-cell and collective contexts and imply that the loss of LKB1 impairs ECM boundary sensing.

LKB1 is necessary for haptotaxis but is not required for chemotaxis to PDGF

Based on the observation that migrating cells with LKB1 displayed a "pausing" at the wound boundary, we postulated that cells with LKB1 could recognize ECM content on the surface but that LKB1-null cells were refractory to this cue. To test this hypothesis, we used microfluidic chamber devices in which we previously demonstrated an ability to generate stable linear gradients of immobilized ECM (Fig. 3 A; Wu et al., 2012). This approach allows visualization of directional cell migration toward ECM (haptotaxis) for time periods of $\leq 24 \mathrm{~h}$. We mixed tdTomato-labeled LKB1-null cells and GFP-labeled addback cells and observed their haptotactic migration by time-lapse microscopy in the chamber. We quantified three independent parameters to describe overall migration behavior: velocity; the forward migration index (FMI; Monypenny et al., 2009), the distance migrated in the direction of the gradient over the total accumulated distance; and persistence, the displacement over the total path length. We considered haptotaxing cells as those with a mean FMI and a 95\% confidence interval $>0$. Cells that did not haptotax had a mean FMI and a 95\% confidence interval encompassing 0 . To our surprise, we found that cells with intact LKB1 (addback) migrated directionally toward the gradient, whereas cells without LKB1 had no directional preference (Fig. 3 B). We confirmed this observation in LKB878 LKB1null/addback cells (Fig. S2 A) as well as in TKL2 cells (Fig. S2 B), which had a significantly enhanced ability to haptotax upon LKB1 addback. Furthermore, to determine whether the loss of LKB 1 could abolish haptotactic capacity in other mesenchymal cells, we depleted LKB1 in IA32 mouse embryonic fibroblasts (Fig. S2 C; Cai et al., 2008). Similar to our results using melanoma cells, fibroblasts depleted of LKB1 failed to haptotax in contrast to their control counterparts (Fig. S2, D and E).

To test whether proximal integrin signaling was still intact, we held LKB1-null and addback melanoma cells in suspension for $3 \mathrm{~h}$ and subsequently plated them for $1 \mathrm{~h}$ on fibronectincoated surfaces (Fig. 3 C). Loss of integrin engagement resulted in reduced phosphorylation of FAK at tyrosine 397, which was restored in cells in both null and addback cells upon adhesion. Together, these data indicate that the loss of LKB1 does not affect the recognition of absolute concentrations of ECM but ablates the ability to distinguish a gradient of ECM across a single cell.

To confirm our findings in a 3D migration environment, we used microfluidics chambers to generate a 3D ECM gradient. To accomplish this, we seeded cells in 3D collagen gels and established a linear gradient of fibronectin within the collagen gel by diffusion (Fig. 3 D). By confocal microscopy, we observed that the Cy5-fibronectin became associated with collagen fibrils in a concentration gradient from one side of the chamber to the other. Once this immobilized gradient formed, we flushed the soluble Cy5-fibronectin out of the source channel and observed a stable gradient, confirming that this fibronectin was bound to the collagen in the gel. In this configuration, we observed a similar difference in haptotactic capacity between LKB1-null and addback cells as in 2D, whereby the null cells were unable to sense and/or respond to the fibronectin gradient (Fig. 3 E). These results further support the notion that loss of LKB1 abolishes directional migration toward ECM gradients.

One possible explanation for this difference in haptotactic migration is that the presence or absence of LKB1 affects a nonautonomous property of the cells. For example, LKB1 loss could promote enhanced secretion of matrix metalloproteinases (MMPs; Ou et al., 2012). MMPs could ostensibly cause the degradation of matrix surrounding the cell, rendering it unable to sense an ECM gradient. Because all of the 2D and 3D haptotaxis experiments described in this paper used populations of LKB1-null and addback cells mixed together in the same chamber (with differential fluorescent marking), we do not think that a nonautonomous effect is a likely explanation for the difference. However, to test whether invasion into $3 \mathrm{D}$ collagen was

null, $n=28$; addback, $n=23$.) (D) Single-cell speed of LKB498 cells on increasing fibronectin concentrations. $(0.1 \mu \mathrm{g} / \mathrm{ml}: \mathrm{null}, n=94 ;$ addback, $n=104.1 \mathrm{gg} / \mathrm{ml}$ : null, $n=99$; addback, $n=96.10 \mu \mathrm{g} / \mathrm{ml}$ : null, $n=149$; addback, $n=162.100 \mu \mathrm{g} / \mathrm{ml}:$ null, $n=134 ;$ addback, $n=150$.) (E) Montage images and edge outlines of time-lapse scratch wounds. (F) Relative wound area over time. (LKB498: null, $n=43 ;$ addback, $n=32$. TKL2: null, $n=33$; addback, $n=33$.) Gray shaded area indicates times at which relative wound area shows significant differences with $P<0.01$. Insets show magnified plots of time points at which pausing occurs (represented by the bars) at the wound boundary. (G) Scratch wound of LKB498 LKB1 addback monolayer on Cy3-fibronectin (FN). DIC, differential interference contrast. All data are shown as means $\pm \mathrm{SEM}$. ${ }^{*}, \mathrm{P}<0.05 ; * *, \mathrm{P}<0.01 ; * * *, P<0.001 ; * * * *, P<0.0001$ by two-tailed unpaired $t$ test. Bars, $100 \mu \mathrm{m}$. 

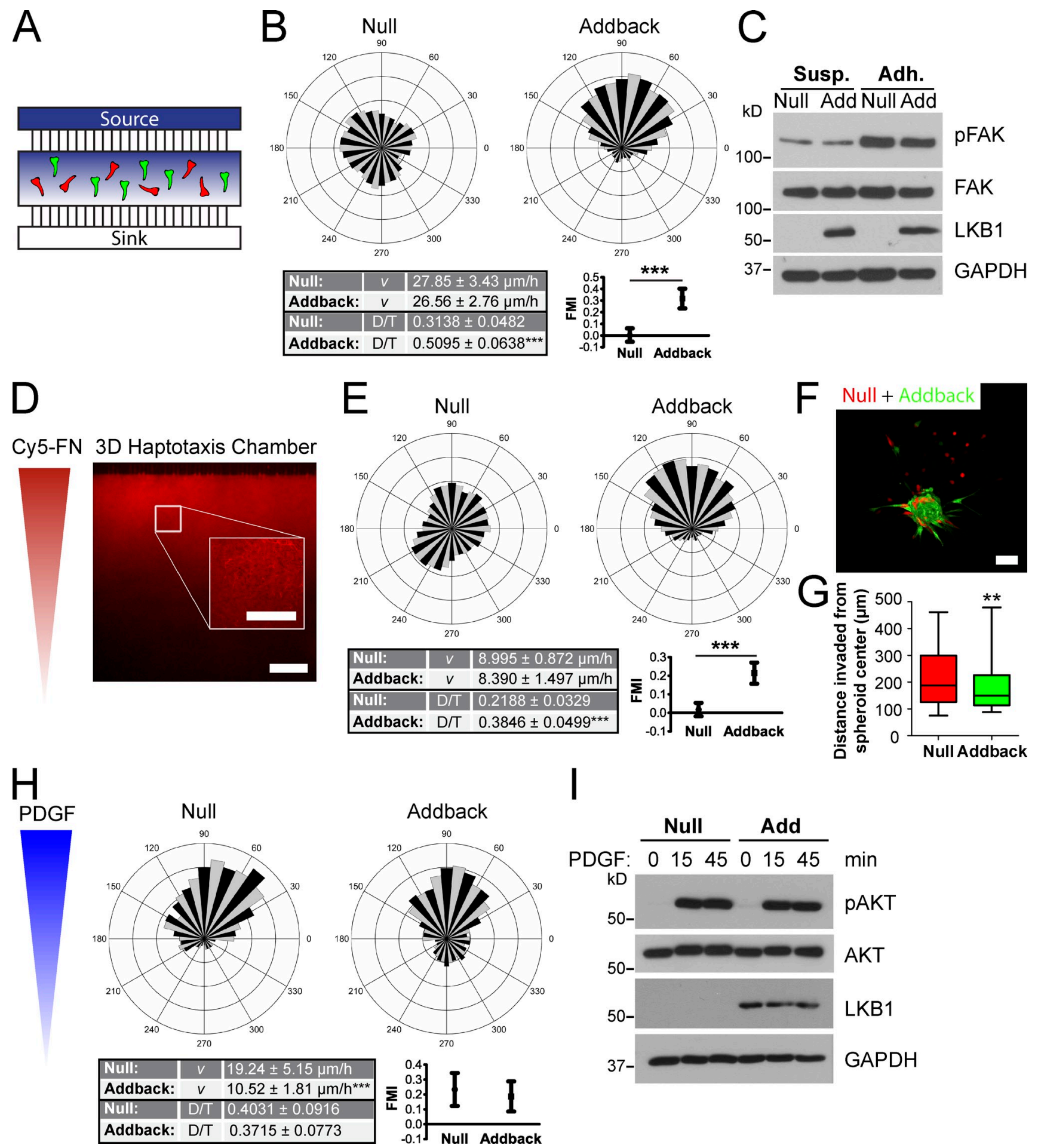

Figure 3. LKB1 is necessary for haptotaxis but is not required for directional migration to PDGF. (A) Schematic of mixed fluorescent cells in microfluidics chamber system. (B) LKB1 is required for haptotaxis of LKB498 cells to fibronectin. (null, $n=99$; addback, $n=75$.) (C) Western blots showing phosphorylation of FAK in LKB498 cells upon suspension (Susp.) or adhesion (Adh.) to fibronectin. Add, addback. (D) Epifluorescence image of 3D haptotaxis chamber with Alexa Fluor 647-fibronectin (FN) gradient across $1 \mathrm{mg} / \mathrm{ml}$ 3D collagen. Inset shows a confocal image of fibronectin bound to collagen fibrils. (E) LKB 1 is necessary for haptotaxis of LKB498 cells in 3D collagen. (null, $n=132$; addback, $n=89$.) (F and G) Spheroid invasion of TKL2 cells into 3D collagen is cell autonomous. (null, $n=118$; addback, $n=120$.) Data are shown as a box and whisker plots with the boxes extending from the 25th through 75 th percentiles with the lines in the middle as the medians. Whiskers range from the minimum to maximum values. ${ }^{*}, \mathrm{P}<0.01$ by two-tailed unpaired $t$ test. $(\mathrm{H}) \mathrm{LKB} 1$ is dispensable for chemotaxis of LKB498 cells to PDGF. (null, $n=34$; addback, $n=39$.) (I) Western blots showing phosphorylation of AKT upon acute stimulation with $200 \mathrm{ng} / \mathrm{ml} \mathrm{PDGF}$. All haptotaxis and chemotaxis data are shown as mean $\pm 95 \%$ confidence intervals. ${ }^{* *}, \mathrm{P}<0.001$ by two-tailed unpaired $t$ test. Bars, $100 \mu \mathrm{m} . v$, velocity; D/T, displacement (D) over the total path length (T). 

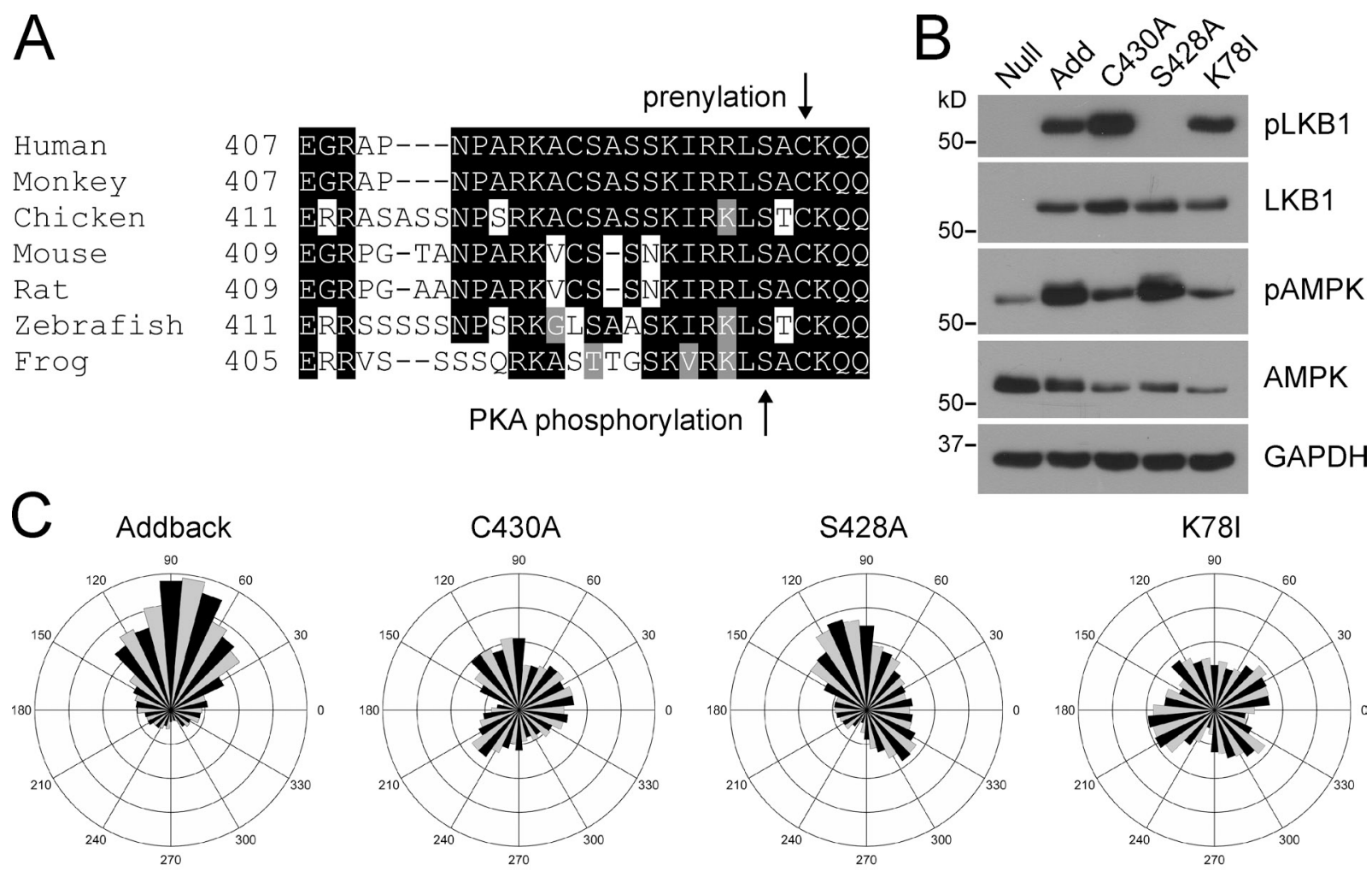

GAPDH

\begin{tabular}{|l|c|c|}
\hline Addback & \multicolumn{1}{c}{$v$} & D $/ \mathrm{T}$ \\
\hline WT: & $18.99 \pm 2.69 \mu \mathrm{m} / \mathrm{h}$ & $0.4282 \pm 0.0708$ \\
\hline C430A: & $23.82 \pm 3.72 \mu \mathrm{m} / \mathrm{h}^{*}$ & $0.4022 \pm 0.0648$ \\
\hline WT: & $22.95 \pm 2.88 \mu \mathrm{m} / \mathrm{h}$ & $0.4555 \pm 0.0422$ \\
\hline S428A: & $22.78 \pm 2.98 \mu \mathrm{m} / \mathrm{h}$ & $0.4175 \pm 0.0607$ \\
\hline WT: & $30.89 \pm 3.51 \mu \mathrm{m} / \mathrm{h}$ & $0.5404 \pm 0.0617$ \\
\hline K78I: & $35.51 \pm 3.45 \mu \mathrm{m} / \mathrm{h}$ & $0.4368 \pm 0.0753^{*}$ \\
\hline
\end{tabular}

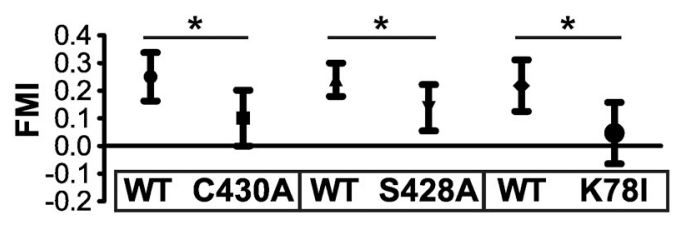

Figure 4. Membrane targeting and kinase activity of LKB1 are required for haptotaxis. (A) Sequence alignment of vertebrate LKB $1 \mathrm{C}$ terminus. Black shade indicates identical amino acids, and gray shade indicates similar amino acids. (B) Western blots of LKB 1 reconstitution in LKB498 cells with wild type (WT) or prenylation (C430A), phosphorylation (S428A), and kinase-dead (K78I) mutants. Add, addback. (C) Membrane association and kinase activity are required for haptotaxis. (addback, $n=52 ; \mathrm{C} 430 \mathrm{~A}, n=37 ; \mathrm{S} 428 \mathrm{~A}, n=68 ; \mathrm{K} 78 \mathrm{I}, n=42$.) All data are shown as mean $\pm 95 \%$ confidence intervals. $*, P<0.05$ by two-tailed unpaired $t$ test. $v$, velocity; $D / T$, displacement $(D)$ over the total path length (T).

cell autonomous, we examined spheroid invasion with mixed fluorescent LKB1-null and addback cells (Fig. 3 F). Despite the fact that the cells were in close contact for many hours, the majority of cells invaded cell autonomously with LKB1-null cells migrating a farther distance as compared with addback cells (Fig. 3 G). Moreover, to formally test whether MMP inhibition was able to restore haptotaxis in LKB1-null cells, we performed haptotaxis experiments in the presence of the MMP inhibitor GM6001, used at concentrations that were sufficient to block invadopodial matrix degradation in A2058 cells (Fig. S2 F), both in 2D (Fig. S2 G) and in 3D (Fig. S2 H) configurations. We found no deviation from our results obtained in the absence of MMP inhibition, demonstrating that the difference in haptotaxis between LKB1-null and addback cells is not MMP dependent.

Several studies have implicated the role of LKB1 in maintaining cell polarity (Asada et al., 2007; Rodríguez-Fraticelli et al., 2012). Therefore, it is possible that loss of LKB1 impairs all forms of directional migration. To investigate this possibility, we used microfluidics chambers to generate a soluble gradient of PDGF, a potent chemotactic ligand for melanoma cells, and assessed directional migration toward this cue. Remarkably, regardless of LKB1 status, cells efficiently chemotaxed to PDGF (Fig. $3 \mathrm{H}$ ). Consistent with this, LKB1 depletion in fibroblasts had no effect on chemotaxis to PDGF (unpublished data). We confirmed that downstream signaling of the PDGF receptor was intact in LKB1-null and addback melanoma cells by measuring AKT phosphorylation upon acute PDGF stimulation (Fig. 3 I). These data demonstrate that the loss of LKB1 specifically ablates directional migration toward ECM gradients but not toward soluble growth factor cues.

\section{Membrane targeting and kinase activity are required for haptotaxis}

LKB1 contains a highly conserved $\mathrm{C}$ terminus that is thought to play an integral role in its regulation (Fig. 4 A). In particular, LKB1 has a CAAX prenylation motif that mediates its membrane association and an upstream PKA/p90RSK phosphorylation site that has been shown to regulate cell polarity (Martin and St Johnston, 2003; Barnes et al., 2007; Shelly et al., 2007). To determine whether these posttranslational modifications contribute 
to LKB1's regulation of haptotaxis, we reconstituted LKB1-null melanoma cells with mutant forms of LKB1 that were resistant to modification. We introduced a C430A mutation, which abolishes LKB1 prenylation and is predicted to prevent association with the plasma membrane (Collins et al., 2000). We also made a S428A mutation, which prevents cAMP and extracellular signal-related kinase-mediated PKA/p90RSK phosphorylation at this site (Sapkota et al., 2001). Additionally, to test whether kinase activity was required, we reconstituted cells with a kinasedead mutant (K78I) of LKB1. We verified that all the LKB1 mutants had equal levels of expression (Fig. 4 B). Interestingly, phosphorylation of S428 was increased in the prenylationdeficient C430A mutant, indicating a possible feedback between phosphorylation and membrane targeting. Both cells expressing wild-type LKB1 or the S428A mutant displayed enhanced phosphorylation of AMPK. This corresponded with their ability to haptotax toward a fibronectin gradient (Fig. 4 C). Interestingly, although we found that PKA/p90RSK phosphorylation was not required for haptotaxis per se, haptotactic fidelity was significantly diminished. Cells expressing the C430A or the K78I mutant showed impaired AMPK phosphorylation, and they could not haptotax. Our finding that AMPK phosphorylation was reduced with the $\mathrm{C} 430 \mathrm{~A}$ mutant is also consistent with data reported from the recent generation of a mutant LKB1 knock-in mouse (Houde et al., 2014). Together, these results demonstrate that membrane association and kinase activity of LKB1 are required for haptotaxis.

MARK family kinases are the key LKB1 substrates necessary for haptotaxis

The requirement for LKB1 kinase activity in governing haptotaxis prompted us to next ask which downstream substrate of LKB1 is necessary. LKB1 is a master regulatory kinase that phosphorylates 14 different kinases and activates all of them with the exception of maternal embryonic leucine zipper kinase (Lizcano et al., 2004). To determine which LKB1 substrates are expressed, we performed RNA-Seq (RNA sequencing) transcriptome analysis of LKB498-null and addback cells. Of the known LKB1-activated kinase substrates, mRNA for nine of them was expressed: Ampk1, Brsk1, Nuak1, Sik1, Sik2, Sik3, Mark2, Mark3, and Mark4 (Fig. S3 A). Of note, we did not find dramatic transcriptional changes in any of these upon LKB1 reintroduction (Table $\mathrm{S} 1$ ).

To test the importance of each LKB1 substrate in haptotaxis, we used lentiviral shRNA knockdown and pharmacological inhibition. We first tested the requirement for AMPK, the major LKB1 substrate involved in cell metabolism that inhibits mammalian target of rapamycin (Shaw et al., 2004a) by using multiple lentiviral shRNAs to knock down AMPK (Fig. S3 B). Interestingly, we found that LKB1-mediated haptotaxis does not require AMPK (Fig. S3 L). In addition, lentiviral knockdown of BRSK1, which plays a key role in neuronal polarization, demonstrated that it is dispensable for haptotaxis (Fig. S3, C and L; Barnes et al., 2007). Given that the nua kinase (NUAK) family kinases are known to inhibit the activity of myosin phosphatase to control cell adhesion and are a promising candidate to regulate directional cell migration (Zagórska et al., 2010), we also tested the requirement of NUAK1 by shRNA knockdown and found no effect on haptotaxis (Fig. S3, D, E, and L). As an additional approach, we used the recently described selective inhibitor of NUAK family kinases WZ4003 (Banerjee et al., 2014). We confirmed that the NUAK inhibitor decreased myosin light chain phosphorylation at serine 19 in a dose-dependent manner (Fig. S3, F and G); however, similar to NUAK1 knockdown, LKB1 addback cells treated with the NUAK inhibitor could still haptotax (Fig. S3 H). Finally, we simultaneously depleted all isoforms of the SIK family kinases and found no effect on haptotaxis (Fig. S3, I-L). Collectively, these data indicate that the LKB1 effectors AMPK, BRSK, NUAK, and SIK are not required for haptotaxis (Figs. $5 \mathrm{~A}$ and S3 L).

The MARK family kinases (Drewes et al., 1997) are mammalian orthologues of the C. elegans Par-1 (Guo and Kemphues, 1995), which play key roles in regulating cell polarity and are, therefore, attractive targets that may regulate directional migration (Sapir et al., 2008; Mejia-Gervacio et al., 2012). To determine whether MARK family kinases are necessary for haptotaxis, we depleted all the expressed isoforms (MARK2, MARK3, and MARK4) in LKB1 addback melanoma cells (Fig. S4, A and B). In contrast to the other LKB1 effectors, we found that MARK family kinases are essential for haptotaxis (Fig. 5 B).

We next asked whether MARK family kinase activity could regulate haptotactic migration. A primary function of the MARK family kinases is to phosphorylate the microtubuleassociated proteins MAP4, MAP2, and Tau (Illenberger et al., 1996). According to our RNA-Seq data, the predominant microtubule-associated protein expressed in melanoma cells is MAP4. By Western blotting, we detected MAP4 ( 220 kD; Chapin et al., 1995) and phosphorylated MAP4 ( 121 kD), which was enhanced in addback as compared with null cells (Fig. 5 C). The recently described MARK inhibitor 39621 (half-maximal inhibitory concentration $=3.6 \mu \mathrm{M}$ ) is an ATP-competitive and highly selective MARK family kinase inhibitor that has minimal effects on other AMPK-related kinases (Timm et al., 2011). We verified that the MARK inhibitor was functional by its ability to block MAP4 phosphorylation in both LKB1-null and addback cells (Fig. 5 C). To determine whether MARK activity is required for haptotaxis, we treated LKB1 addback cells with the MARK inhibitor. Consistent with our hypothesis, treatment with the MARK inhibitor abrogated haptotaxis (Fig. 5 D). We substantiated this observation using fibroblasts treated with the MARK inhibitor using a before and after a wash-in regimen in our microfluidic chambers (Fig. S4 C).

As a complementary approach to treatment with MARK inhibitor, we overexpressed a known MARK3-T211A/S215A mutant that LKB1 and MARK kinase cannot phosphorylate/activate (Marx et al., 2010). We confirmed its function as a dominantnegative (DN; DN-MARK3) by its ability to completely abrogate endogenous MAP4 phosphorylation, which was even lower than we observed in LKB1-null cells (Fig. 5 E). Overexpression of DN-MARK3 in LKB1 addback cells abolished haptotaxis both in 2D (Fig. 5 F) and 3D (Fig. 5 G). Collectively, these data demonstrate that MARK family kinases are the major LKB1 substrates that are required for haptotaxis. 
A

\begin{tabular}{|l|l|l|}
\hline Substrate & Expressed? & $\begin{array}{l}\text { Required for } \\
\text { haptotaxis? }\end{array}$ \\
\hline AMPK1 & Yes & No \\
\hline AMPK2 & No & N/A \\
\hline BRSK1 & Yes & No \\
\hline BRSK2 & No & N/A \\
\hline NUAK1 & Yes & No \\
\hline NUAK2 & No & N/A \\
\hline SIK1 & Yes & No \\
\hline SIK2 & Yes & No \\
\hline SIK3 & Yes & No \\
\hline
\end{tabular}
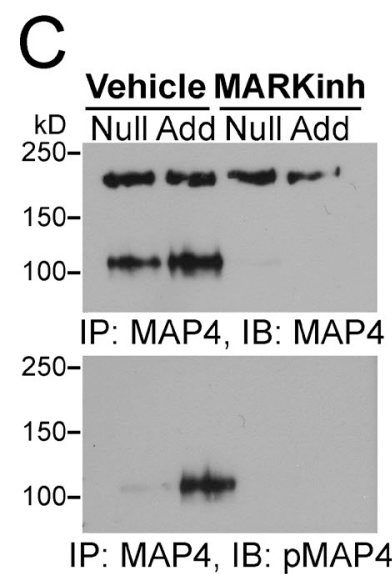

F Addback

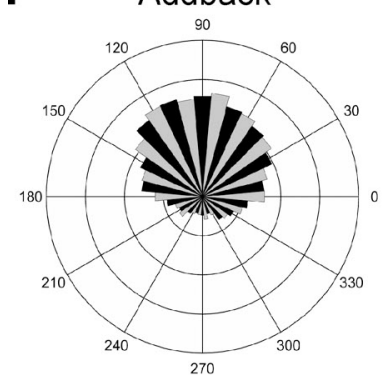

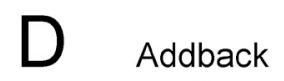
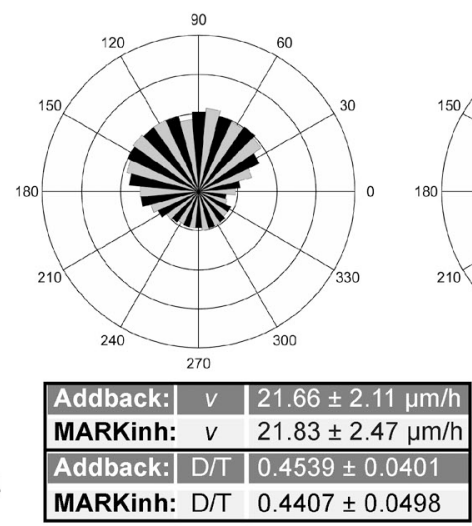

B Addback
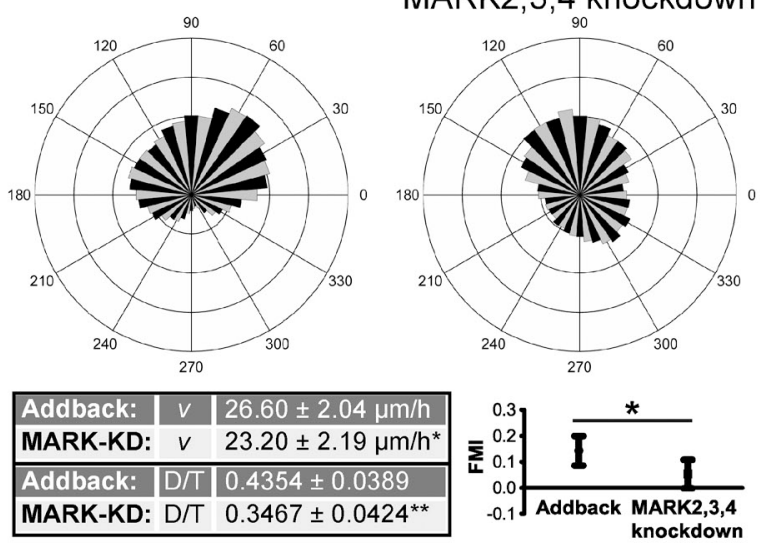

Addback +

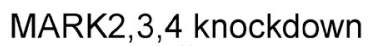

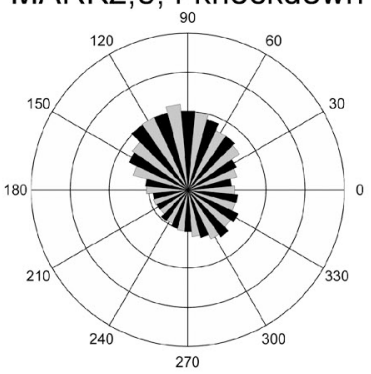
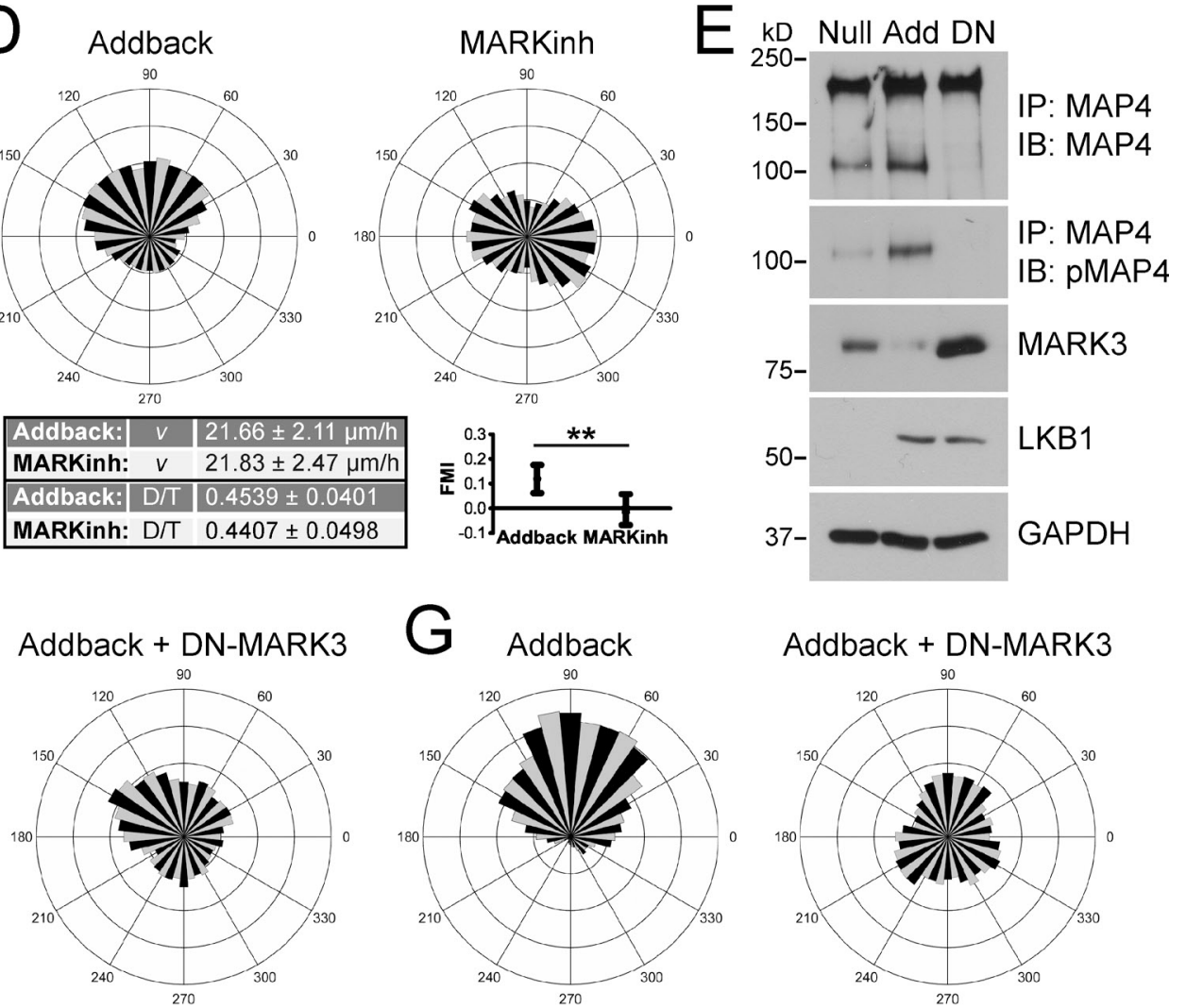

Addback + DN-MARK3
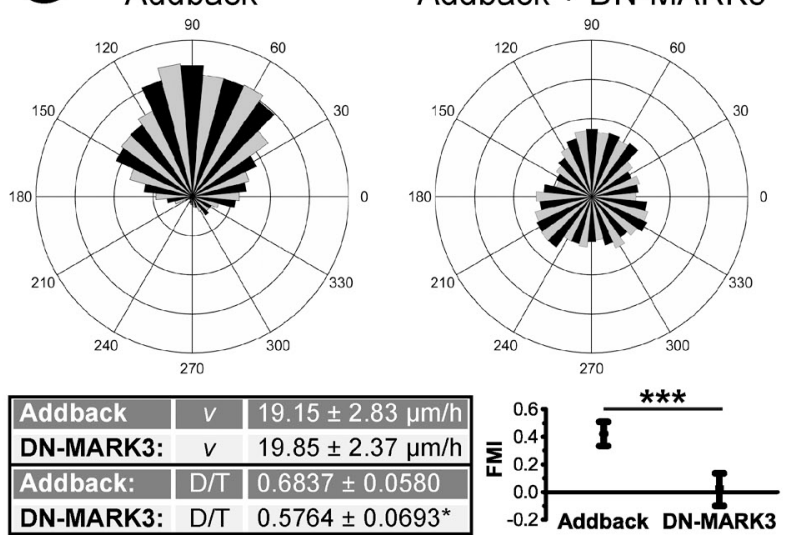

Figure 5. MARK family kinase activity is necessary for haptotaxis. (A) Several LKB 1 substrates are not required for haptotaxis. Substrates not expressed were not tested in haptotaxis experiments and are listed as not available (N/A). (B) MARK family kinases are required for haptotaxis in LKB498 cells. (addback, $n=125$; addback + MARK2, 3, and 4 knockdown [KD], $n=106$.) (C) Western blots showing MARK inhibitor (MARKinh) 39621 (20 $\mu$ M) validation in LKB498 cells. Add, addback; IP, immunoprecipitation; IB, immunoblot. (D) MARK inhibitor blocks haptotaxis in LKB498 cells. (addback, $n=$ 133; MARK inhibitor, $n=92$.) (E) Western blots validating effect of dominant-negative (DN) MARK3 overexpression in LKB498 cells. (F and G) DN-MARK3 blocks haptotaxis of LKB498 cells in 2D (F) and 3D (G). (2D: addback, $n=130 ;$ DN-MARK3, $n=131$. 3D: addback, $n=48 ;$ DN-MARK3, $n=40$.) All data are shown as mean $\pm 95 \%$ confidence intervals. ${ }^{*}, \mathrm{P}<0.05 ;{ }^{*}, \mathrm{P}<0.01 ; * * *, \mathrm{P}<0.001$ by two-tailed unpaired $t$ test. $v$, velocity; $\mathrm{D} / \mathrm{T}$, displacement (D) over the total path length (T).

Haptotaxis does not require MAP4

or microtubules

MARK phosphorylation of MAP4 on KXGS repeats 1 and 4 induces its dissociation from microtubules and subsequently results in microtubule destabilization (Illenberger et al., 1996). Based on previous studies showing microtubule involvement in the regulation of cell polarity and directional migration (Magdalena et al., 2003; Xu et al., 2005), we hypothesized that 

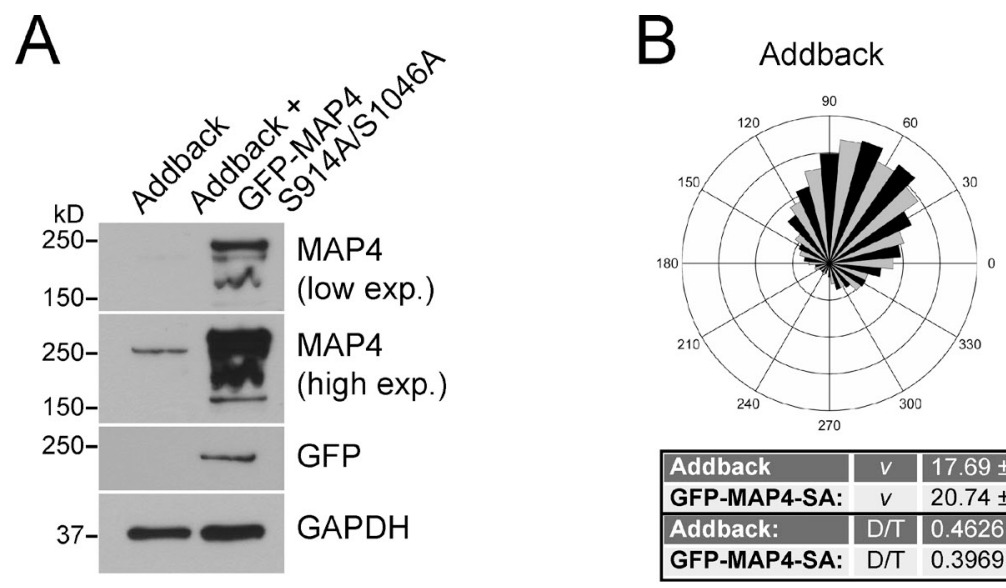

Addback +

GFP-MAP4-S914A/S1046A

C

Addback
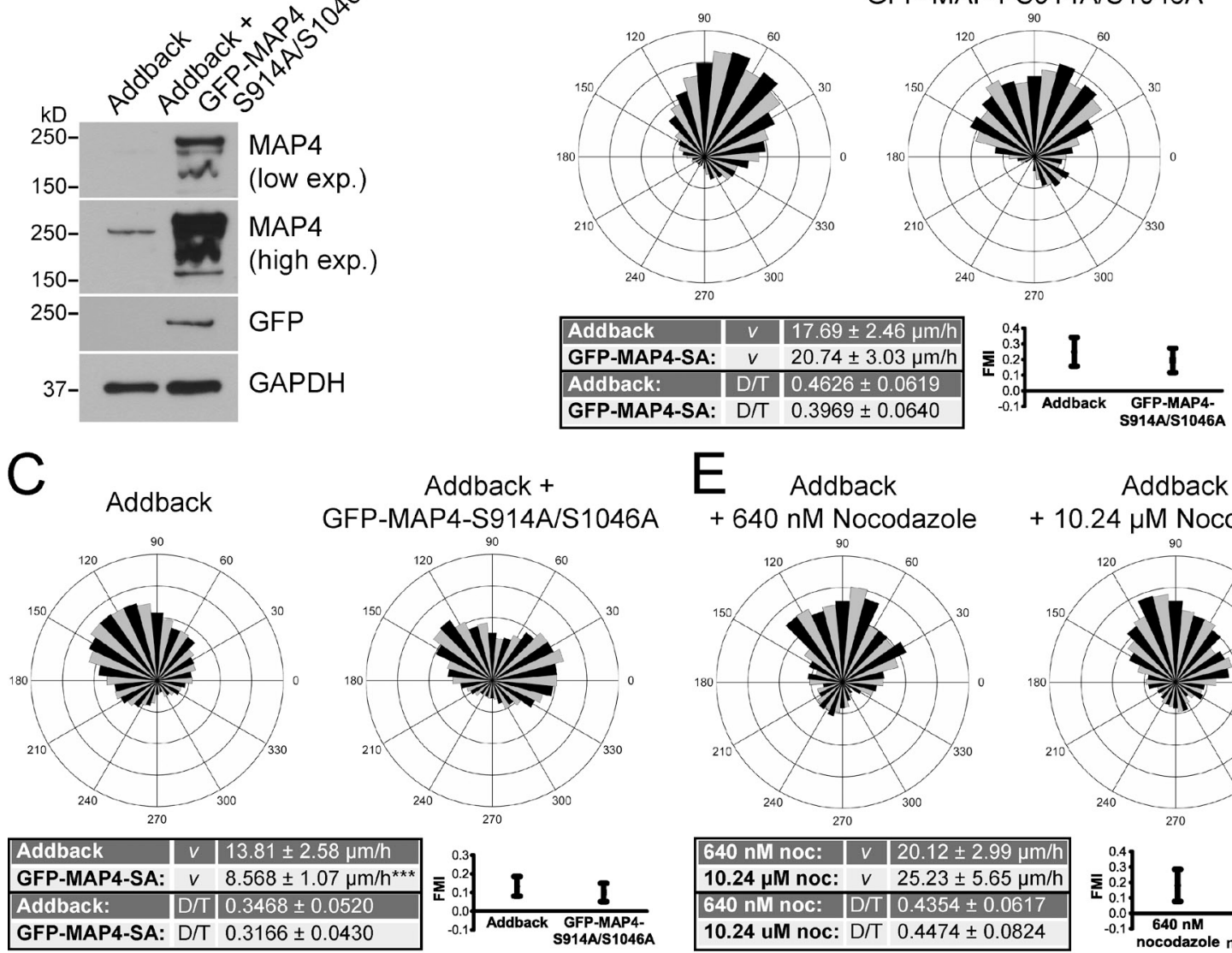

Addback
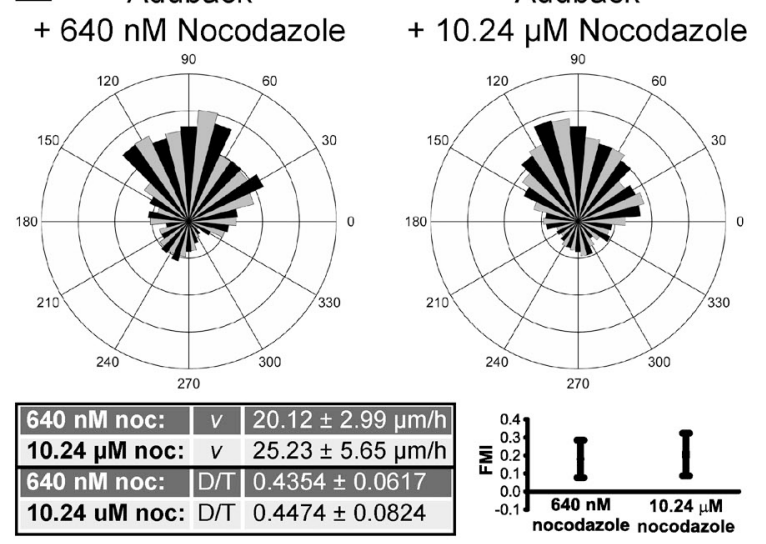

$D$

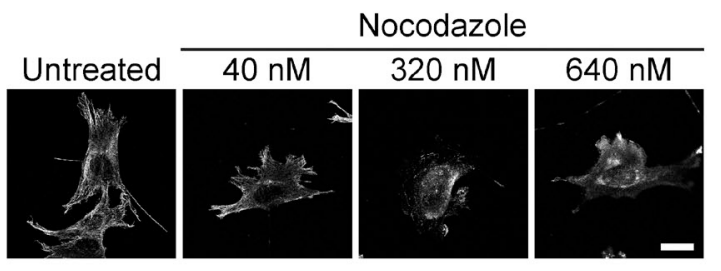

F Before Wash-in

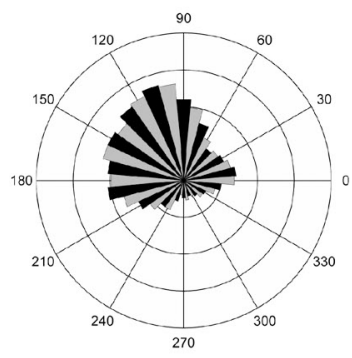

\begin{tabular}{|l|l|l|}
\hline Before: & $v$ & $13.80 \pm 1.76 \mu \mathrm{m} / \mathrm{h}$ \\
\hline
\end{tabular}

Wash-in: $v \quad 22.40 \pm 5.92 \mu \mathrm{m} / \mathrm{h}^{\star \star}$ \begin{tabular}{l|l|l}
\hline Before: & $\mathrm{D} / \mathrm{T}$ & $0.4337 \pm 0.0631$
\end{tabular}

Wash-in: $\mathrm{D} / \mathrm{T} \quad 0.3232 \pm 0.0649^{* *}$
$10 \mu \mathrm{M}$ Nocodazole Wash-in
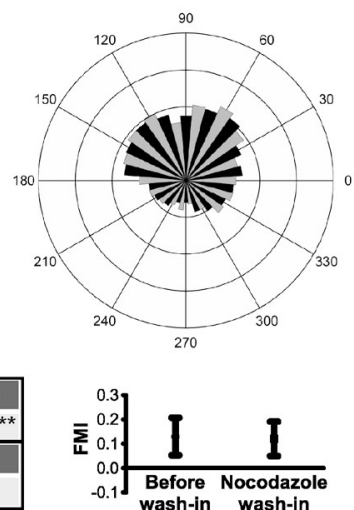

$G$ Before Wash-in
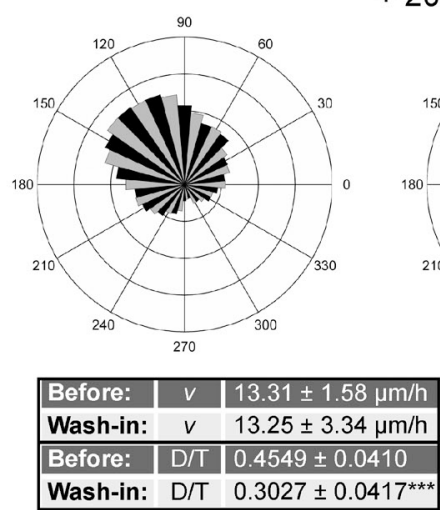

$10 \mu \mathrm{M}$ Nocodazole + $20 \mu$ M MARKinh Wash-in
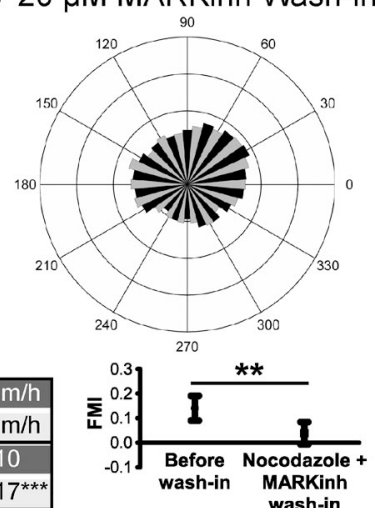

Figure 6. Haptotaxis does not require MAP4 or microtubules. (A) Western blots showing overexpression of active MAP4 in LKB498 cells. exp., exposure. (B and C) Active MAP4 does not affect haptotaxis of LKB498 cells in 2D (B) and 3D (C). (addback, $n=51$; GFP-MAP4-S914A/S1046A [SA], $n=48$.) (D) Nocodazole depolymerizes microtubules in LKB498 cells. Bar, 20 mm. (E) Nocodazole (noc) treatment of LKB498 LKB1 addback cells has no effect on haptotaxis. (640 $\mathrm{nM}, n=37 ; 10.24 \mu \mathrm{M}, n=35$.) ( $\mathrm{F}$ and $\mathrm{G}$ ) Washing of nocodazole alone (F) does not block haptotaxis (before wash in, $n=69 ;$ wash in, $n=51$ ) but together with MARK inhibitor (G) blocks haptotaxis in IA32 fibroblasts. (before wash in, $n=155$; wash in, $n=107$.) All haptotaxis data are shown as mean $\pm 95 \%$ confidence intervals. ${ }^{* *}, \mathrm{P}<0.01 ;{ }^{* *}, \mathrm{P}<0.001$ by two-tailed unpaired $t$ test. $v$, velocity; $\mathrm{D} / \mathrm{T}$, displacement (D) over the total path length (T). 
MARK phosphorylation of MAP4 would be the key mechanism upstream of microtubules to regulate haptotaxis. To test this, we generated a MAP4 mutant GFP-MAP4-S914A/S1046A, which prevents MARK family kinase phosphorylation of MAP4 and promotes microtubule assembly (Chinnakkannu et al., 2010). We overexpressed GFP-MAP4-S914A/S1046A in LKB1 addback cells (Fig. $6 \mathrm{~A}$ ) and tested their ability to haptotax. Unexpectedly, overexpression of mutant MAP4 in LKB1 addback cells had no effect on haptotaxis in 2D (Fig. 6 B) and 3D (Fig. 6 C). This result suggests that MARK regulates haptotaxis in a manner independent of its ability to affect microtubules through MAP4 phosphorylation.

We next investigated the role of microtubules in the process of haptotaxis by perturbing microtubule dynamics upon treatment of cells with nocodazole. We determined that microtubules were completely depolymerized at a nocodazole concentration of $640 \mathrm{nM}$ (Fig. $6 \mathrm{D}$ ). We then evaluated the ability of LKB1 addback cells to haptotax in the presence of nocodazole at this concentration (Fig. 6 E). To our surprise, despite clear alteration in cellular morphology during migration, nocodazole had no discernable effect on haptotaxis. To further support this, we used a 16-fold higher dose and found the same result. Likewise, in fibroblasts, we performed nocodazole wash in and found no effect on their ability to haptotax (Fig. 6 F). However, in accordance with our previous finding, wash in of nocodazole together with MARK inhibitor blocked haptotaxis (Fig. $6 \mathrm{G}$ ). Collectively, these data highlight the striking finding that MARK regulates haptotaxis in a manner that does not depend on MAP4 or intact microtubules.

\section{MARK family kinases limit cell invasion}

To determine whether the loss of haptotaxis as a result of the inhibition of MARK function also affects 3D invasion, we prepared spheroids of LKB498 LKB1 addback cells overexpressing DN-MARK3 and embedded them into collagen (Fig. S5 A). Overexpression of DN-MARK3 enhanced invasive outgrowth in cells with intact LKB1 (Fig. S5 B). Furthermore, treatment of LKB1 addback cells with the MARK inhibitor enhanced invasion into 3D collagen (Fig. S5 C). To confirm this observation in another LKB1-deficient melanoma cell line derived from the same genetic model, we generated TKL2 cells depleted of endogenous MARK3 and overexpressed DN-MARK3 (Fig. 7 A). Importantly, overexpression of DN-MARK3 had no effect on AMPK phosphorylation (Fig. 7 B). Similar to our observation in LKB498 cells, MARK disruption in TKL2 cells enhanced spheroid invasion into 3D collagen (Fig. 7, C and D). These findings indicate that MARK family kinases, substrates of LKB1 that are required for haptotaxis, limit melanoma invasion.

\section{iscussion}

In this work, we discovered a hitherto undescribed role for an LKB1-MARK axis as a mediator of directional migration toward gradients of ECM. Therefore, we postulate that ECM cues limit cell invasion in LKB1-competent cells. In Ras-driven melanoma, the loss of LKB1 signaling through MARK and loss of the ability to recognize ECM gradients is associated with enhanced

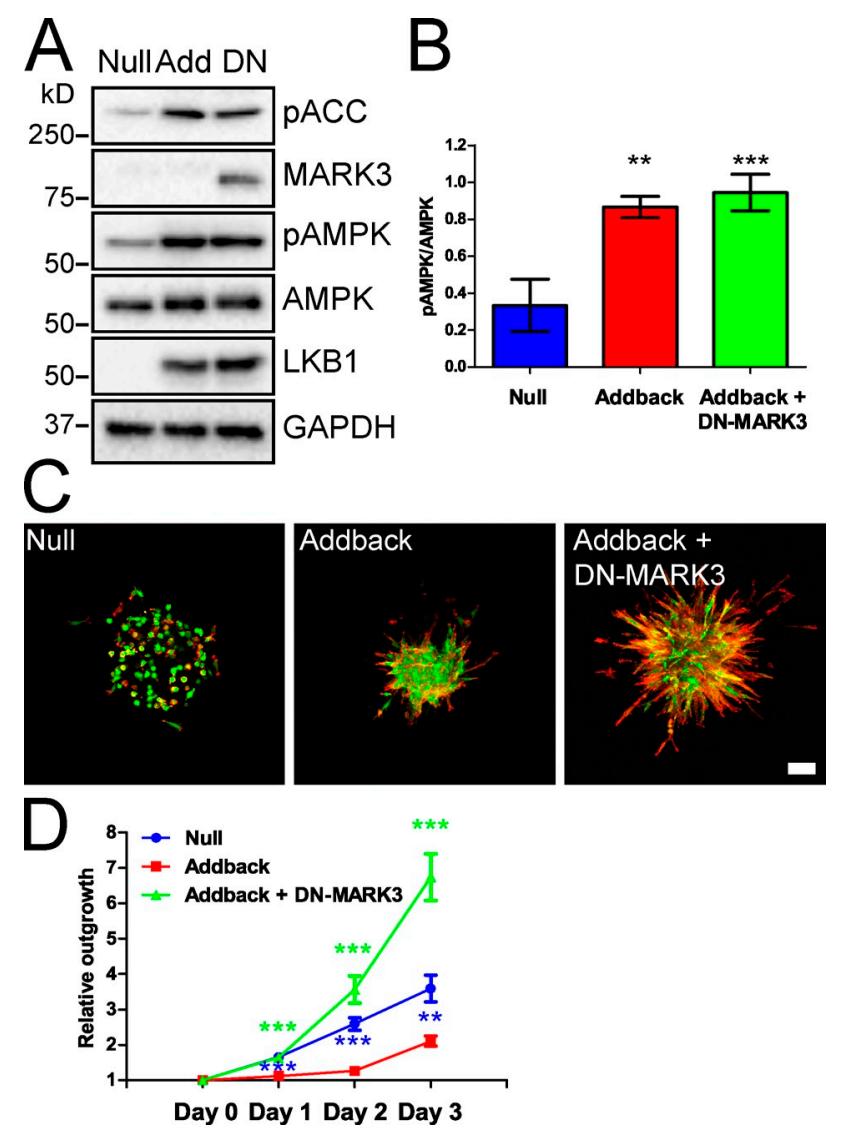

Figure 7. MARK inhibition facilitates cell invasion. (A) Western blot of overexpression of DN-MARK3 in TKL2 cells shows no effect on AMPK phosphorylation. pACC, phosphorylated acetyl-CoA carboxylase. (B) Quantification of Western blot data $(n=3)$ in $A$ is shown as means \pm SEM. $* *, P<0.01 ; * * *, P<0.001$ by two-tailed unpaired $t$ test. (C) $D N$ MARK3 enhances spheroid invasion into $4 \mathrm{mg} / \mathrm{ml} 3 D$ collagen. Cells were stained with Alexa Fluor 568-phalloidin to label actin. Bar, $100 \mu \mathrm{m}$. (D) Quantification of data in C is shown as means \pm SEM. (day 0-3: null, $n=14$; addback, $n=9$; addback + DN-MARK3, $n=22$.) **, $\mathrm{P}<0.01$; $* * *, P<0.001$ by one-way analysis of variance (ANOVA) by Dunnett's post-test, as compared with addback.

invasion. Future studies should investigate whether this applies to metastasis in vivo.

We anticipated that LKB1 loss would facilitate matrix degradation through invadopodia formation to promote invasion. To our surprise, multiple highly metastatic melanoma cell lines did not form invadopodia, regardless of LKB1 status. This does not exclude the possibility that invadopodia can facilitate cell invasion and metastasis; however, our data highlight the loss of haptotaxis as an alternative explanation for how the loss of LKB1 facilitates invasion in melanoma. Many tumors in vivo display a "capsule" of collagen surrounding the tumor periphery, which is thought to result from the outward pushing of preexisting collagen bundles as the tumor proliferates but does not invade this ECM barrier (Hompland et al., 2008). We speculate that the loss of the ability to haptotax caused by LKB1 loss confers a greater degree of plasticity between modes of migration and a failure to respect natural tumor boundaries. Although cells with intact LKB1 can sense and respect ECM boundaries, cells without LKB 1 are free to wander and invade even in the absence of classic invadopodia. 
The processes of tumor cell invasion and, ultimately, metastasis are dependent on the ability of cells to migrate. In multiple contexts, cells that lacked LKB1 still displayed robust frontrear polarity and migrated faster than cells with intact LKB1; however, cells without LKB1 could not haptotax. We previously demonstrated that the Arp2/3 complex and the formation of lamellipodia are necessary for haptotaxis but not chemotaxis to PDGF (Wu et al., 2012). Likewise, cells without LKB1 were unhindered in their ability to chemotax to PDGF, indicating that the loss of haptotaxis is a specific effect in response to ECM cues and does not reflect a general failure of directional migration. Based on these findings, it is tempting to speculate that an LKB1-dependent signaling network is necessary to spatially organize Arp2/3-based lamellipodial protrusions in order for cells to respond to ECM cues. It will be interesting to examine how protrusions are differentially spatially and temporally organized in cells with and without LKB1.

An important open question about LKB1 and its role in cellular signaling is how it is regulated. Studies have suggested that PKA phosphorylation of LKB1 is required for proper neuronal polarization (Barnes et al., 2007; Shelly et al., 2007). However, the recent generation of an LKB1 knock-in mouse that was resistant to phosphorylation at this site showed no overt neurological phenotype, suggesting that there may be contextdependent effects (Houde et al., 2014). In light of these data, we found that cells expressing LKB1-S428A could still haptotax, albeit with less fidelity, suggesting that this phosphorylation site plays a more subtle role in this LKB1 function. On the other hand, membrane targeting and LKB1 kinase activity were strictly required for haptotaxis. Our observation that the LKB1 prenylation mutant was hyperphosphorylated at S428 suggests that the inability to associate with the membrane causes LKB1 to be more susceptible to phosphorylation. It is also possible that phosphorylation facilitates LKB1 membrane association, which could be necessary for optimal haptotactic sensing. Investigation of the interplay between integrin engagement, LKB1 membranetargeting, anddynamic phosphorylationat thePKA/p90RSK site is an area of active ongoing research.

Interaction with the ECM is critical for many, if not most phases, of tumor progression. One hallmark of metastatic dissemination is resistance to apoptosis (Hanahan and Weinberg, 2000). Previously, a kinome-wide loss-of-function screen identified SIK1 as a regulator of p53-dependent anoikis, a specific type of apoptosis caused by loss of integrin-mediated adhesion (Cheng et al., 2009). SIK1 was also necessary for LKB1 to suppress anchorage-independent growth. In the same vein as the loss of SIK1 activity in conferring anoikis resistance, our data demonstrate a connection between the loss of LKB1 and MARK activity and the loss of haptotaxis to facilitate melanoma invasion. Moreover, cells with or without LKB1 displayed biphasic motility and enhanced FAK phosphorylation upon integrinmediated adhesion, suggesting that proximal integrin signaling is still intact. Together with the functional haptotaxis experiments, these data support the notion that LKB1 can translate integrin signaling into a polarized cell response across a gradient. It is likely that LKB1 plays multiple roles in limiting tumor progression in vivo using a variety of mechanisms, such as the expansion of a
CD24 ${ }^{+}$subpopulation (Liu et al., 2012), Src family kinase regulation (Carretero et al., 2010; Liu et al., 2012), cellular metabolism via AMPK (Shaw et al., 2004b), and resistance to anoikis (Cheng et al., 2009). Our data suggest a new mechanism by which LKB1 may limit tumor progression, directly related to invasion and metastasis: loss of LKB1 leads to loss of responsiveness to inhibitory ECM cues.

We found that MARK was the predominant substrate of LKB1 required for haptotaxis. This is intriguing in light of previous data showing the requirement for both LKB1 and MARK in establishing asymmetry in C. elegans (Kemphues et al., 1988). How does MARK confer the ability of cells to undergo haptotaxis? We initially thought that MARK would designate polarity through microtubule regulation by phosphorylating/inactivating MAP4. However, we provide substantial evidence to support the idea that the ability of cells to haptotax is independent of MAP4 and microtubule regulation. It is possible that MARK regulates the actin cytoskeleton by phosphorylating and inhibiting guanine nucleotide exchange factor-H1, a guanine nucleotide exchange factor for RhoA (Yamahashi et al., 2011). Alternatively, a recent genetic screen for kinases that repress the Hippo-Yes-associated protein (YAP) pathway in human cells identified LKB1 and MARK as critical regulators upstream of YAP (Mohseni et al., 2014). Indeed, emerging evidence has established a link between the Hippo-YAP pathway and control of the actin cytoskeleton (Lucas et al., 2013). A more recent study suggests that MARK phosphorylates and localizes the scaffolding protein DIXDC1 to focal adhesions to suppress metastasis in lung cancer (Goodwin et al., 2014). Collectively, these findings highlight an increasingly apparent connection between an ancient, highly conserved polarity pathway and cancer progression.

In summary, we provide substantial evidence that the loss of $\mathrm{LKB} 1$ results in the loss of directional migration toward ECM cues. Our data also support the intriguing hypothesis that the loss of haptotaxis as a result of LKB1 loss facilitates invasion. Future studies will investigate how MARK, the key LKB1 substrate required for haptotaxis, governs invasion in metastatic melanoma.

\section{Materials and methods}

\section{Reagents and materials}

The following commercial antibodies were obtained: rabbit anti-LKB 1 (D60C5), rabbit anti-phospho-LKB1 (Ser428; C67A3), rabbit anti-AMPK$\alpha$ (D5A2), rabbit anti-phospho-AMPK- $\alpha$ (Thr 172; 40H9), rabbit anti-BRSK 1 (D1OF2), rabbit anti-NUAK1/ARK5, rabbit anti-MARK2, rabbit anti-MARK3, rabbit anti-MARK4, and rabbit anti-SIK2 (D28G3; Cell Signaling Technology); rabbit anti-SIK3 (Abgent); mouse anti-GFP (Takara Bio Inc.); rabbit anti-phospho-MAP4 (Ser941; Abnova); rabbit anti-MAP4 (EMD Millipore); and mouse anti-GAPDH (6C5; Life Technologies). Fibronectin was obtained from BD. Alexa Fluor 568-phalloidin, Alexa Fluor 647-phalloidin, and Hoechst 33342 were obtained from Life Technologies. Rat tail collagen type I, PDGF-BB, MARK/Par-1 activity inhibitor 39621, and GM6001 MMP inhibitor were purchased from EMD Millipore.

\section{Cell culture and viral transduction}

A2058 (BRAF $F^{V 600 E} / R B$ null) and WM-266-4 (BRAF ${ }^{V 600 D} / P T E N$ null) human melanoma cell lines were purchased from ATCC. The GR285 (Kras ${ }^{G 12 D} / p 53$ null/p16 null) murine melanoma cell line was derived from 4-hydroxy-tamoxifen (4-OHT)-treated Tyr-Cre-ER $R^{T 2} / \mathrm{Kras}^{\mathrm{ISL}-\mathrm{Gl} 2 \mathrm{D} /+} / \mathrm{p} 53^{\mathrm{L} / L} / \mathrm{pl} 6^{\mathrm{L} / L}$ mice (Monahan et al., 2010). The Tyr-Cre-ERT2 allele is a 4-OHT-inducible Cre recombinase-estrogen receptor fusion transgene under the control 
of the melanocyte-specific tyrosinase promoter (Bosenberg et al., 2006) The Lox-STOP-Lox-Kras ${ }^{\text {G12D }}$ allele harbors a G12D mutation in exon 2 of the endogenous murine Kras gene and contains a STOP cassette flanked by loxP sites that silences the expression of the mutant allele (Jackson et al., 2001). Cre-mediated recombination removes this cassette and allows transcription from the mutated allele. The p $16^{L}$ allele was generated by inserting loxP sites $\sim 3.5 \mathrm{~kb} 5^{\prime}$ to exon $1 \alpha$ and $3^{\prime}$ to the $p 16^{\mathrm{NK} 4 a}$ exon $1 \alpha$, which is excised upon Cre expression and results in a null allele (Monahan et al., 2010). The $p 53^{L}$ allele was generated by inserting loxP sites into Trp53 introns 1 and 10 and has been described previously (Jonkers et al., 2001). Expression of Cre results in a null allele through the deletion of exons 2-10. LKB498, LKB878, and TKL2 (Kras $\left.{ }^{\mathrm{G} 120} / \mathrm{Lkb} 1 \mathrm{null}\right) \mathrm{mu}-$ rine melanoma cell lines were derived from 4-OHT-treated Tyr-Cre-ER ${ }^{T 2} /$ $\mathrm{Kras}^{\mathrm{LSL}-G 12 D /+} / \mathrm{Lkb} T^{L / L}$ mice (Liu et al., 2012). The $L k b l^{L}$ allele contains loxP sites flanking exons 2-6, which are deleted upon Cre-mediated recombination to generate a null allele (Bardeesy et al., 2002). Murine embryonic fibroblasts were described previously (Cai et al., 2008). All cell lines were cultured in DMEM, 10\% FBS, $100 \mathrm{U} / \mathrm{ml}$ penicillin, $100 \mathrm{\mu g} / \mathrm{ml}$ streptomycin, and $292 \mathrm{\mu g} / \mathrm{ml}$ l-glutamine. 293FT cells used for viral packaging were purchased from Life Technologies and transfected using X-tremeGENE 9 (Roche). The shRNAs targeting human and mouse $L K B 1$ in Fig. 1 were designed and cloned into the Hpal-Xhol sites of pLL5.O(GFP) using methods as described previously (Cai et al., 2007). The 19-nt target sequence for human $L K B 1$ for $K D \# 1$ is 5 '-GGGAAGGCTCTTACGGCAA$3^{\prime}$ and KD\#2 is 5'-GAAGAAGGAAATTCAACTG-3'. The target sequence for mouse $l k b l \mathrm{KD \#} 1$ is $5^{\prime}$-GCCAAGCTCATCGGCAAGT-3' and KD\#2 is 5'-GAGAAGCAGAAGATGTATG-3'. The target sequence for the nontargeting shRNA is 5'-GATCGACTTACGACGTTAT-3', which has no exact match in the human, mouse, or rat genome, and has been described previously (Cai et al., 2007). Bulk populations of cells were transduced with lentivirus prepared from these constructs according to standard methods (Cai et al., 2007). GFP-positive cells were sorted by FACS and subjected to invadopodia assays 4-7 d after transduction.

V5-tagged mouse LKB I was cloned into the multicloning site of the pLL5.5 lentiviral vector, which contains an internal ribosome entry site-GFP sequence, or following a GFP/tdTomato-T2A sequence to allow bicistronic expression of GFP/tdTomato and V5-LKB1. To generate LKB1 addback stable melanoma cell lines, bulk populations of LKB498/LKB878/TKL2-null cells were transduced with lentivirus prepared from these constructs and sorted for either GFP or tdTomato expression by FACS. Retroviral transduction of LKBI into bulk populations was performed according to standard methods, and cells were selected for using 2-3 $\mathrm{\mu g} / \mathrm{ml}$ puromycin for $\geq 2-3 \mathrm{~d}$.

The pLKO.1-puro empty vector control and all other shRNAs for mouse Lkb 1, Prkaal (Ampk1), Nuak1, Brsk 1, Sik1, Sik2, Sik3, Mark2, Mark3, and Mark4 were purchased from the University of North Carolina Lenti-shRNA Core Facility, which uses the GE Healthcare TRC1 shRNA libraries. For single shRNA experiments, bulk populations of cells were transduced with lentivirus and selected with puromycin for 2-3 d. Cell lysates were then subjected to Western blotting to assess knockdown efficiency, and cells were assayed for directional migration 4-7 d after transduction. pLKO. 1-hygro (24150) and pLKO.3G (14748; GFP marker) plasmids were purchased from Addgene and used for multiple knockdown experiments. For experiments with knockdown using two shRNAs against the same mRNA to achieve a greater level of knockdown (Lkbl and Nuak 1), cells were simultaneously infected with lentivirus from pLKO.1-puro with one shRNA hairpin and a pLKO. 1-hygro second shRNA hairpin. Cells were then selected with puromycin and $100 \mathrm{\mu g} / \mathrm{ml}$ hygromycin for 4-6 d and tested in directional migration assays 7-10 d after transduction. For triple shRNA knockdowns (Sik and Mark), LKB498 tdTomato-2A-V5-LKB1 cells were simultaneously infected with three lentiviruses with a single shRNA hairpin against each isoform cloned into pLKO.1-puro, pLKO.1-hygro, and pLKO.3G. Cells were selected with puromycin and hygromycin for 6-10 d (longer selection because of lower transduction efficiency), sorted for tdTomato (addback) and GFP (third isoform knockdown) expression by FACS, and tested in haptotaxis assays 10-14 d after transduction. In Fig. 7, to generate TKL2 addback cells depleted of endogenous MARK3 and overexpressing DN-MARK3, bulk populations of TKL2 pBabe.puro-LKB 1 stable addback cells were simultaneously transduced with pBabe.hygro-DN-MARK3 and pLKO.3G-MARK3sh\#06, which targets mouse but not human MARK3. Cells were selected with hygromycin for 4-6 d, and GFP-positive cells were sorted by FACS. A human MARK3 cDNA clone was obtained from the University of North Carolina Tissue Culture Facility, which uses the Human Vidal Orfeome 5.1. The mouse Map4 Mammalian Gene Collection clone was purchased from Life Technologies. LKB 1-K78I was PCR amplified from pBabe. puro-LKB1-K78I and subcloned into pLL5.0-GFP/tdTomato-2A-V5-LKB1
LKB 1-C430A, LKB 1-S428A, MARK3-T2 11 A/S215A (DN-MARK3), and GFP-MAP4-S914A/S1046A (GFP-MAP4-SA) mutants were generated by overlap extension PCR and verified by sequencing.

\section{Western blotting}

Cells were lysed in radioimmunoprecipitation assay buffer $150 \mathrm{mM}$ Tris, $\mathrm{pH} 8.0,1 \% \mathrm{NP}-40,150 \mathrm{mM} \mathrm{NaCl}, 0.5 \%$ sodium deoxycholate, $0.1 \%$ SDS, protease inhibitors, and phosphatase inhibitor cocktail [PhosSTOP; Roche]). Lysates (10-20 $\mathrm{gg}$ total protein) were run on 10\% SDS-PAGE gels and transferred to polyvinylidene fluoride membrane (Immobilon-P; EMD Millipore). Membranes were probed with specific antibodies and HRP-conjugated secondary antibodies (Jackson ImmunoResearch Laboratories, Inc.). Western blots were developed with SuperSignal West Pico or Femto Chemiluminescent Substrate (Thermo Fisher Scientific), scanned on a ChemiDoc MP System (Bio-Rad Laboratories), and quantified using Image Lab 5.0 software.

\section{Invadopodia assay}

Alexa Fluor 405-, Alexa Fluor 568-, and Alexa Fluor 647-gelatin were prepared using the corresponding protein-labeling kits (Life Technologies). Gelatin-coated coverslips were prepared as previously described (Artym et al., 2006). In brief, acid-washed coverslips were coated with $100 \mu \mathrm{g} / \mathrm{ml}$ poly--Llysine in PBS for 20 min, washed in PBS, and subsequently incubated with $0.5 \%$ glutaraldehyde for $15 \mathrm{~min}$. Coverslips were then washed, inverted onto an $80-\mu l$ drop containing an $8: 1 \mathrm{mix}$ of unlabeled $2 \mathrm{mg} / \mathrm{ml}$ gelatin/fluorescently labeled gelatin, and allowed to incubate for $10 \mathrm{~min}$. Coverslips were quenched with $5 \mathrm{mg} / \mathrm{ml}$ sodium borohydride for $15 \mathrm{~min}$ and washed extensively before cell culture.

\section{Live-cell invadopodia assay}

Glass-bottomed dishes (MatTek Corporation) were coated with Alexa Fluor $568-$ gelatin. Cells were plated $8-12 \mathrm{~h}$ before imaging at $37^{\circ} \mathrm{C}$ and $5 \%$ $\mathrm{CO}_{2}$ on an incubator fluorescent microscope (VivaView $\mathrm{FL}$; Olympus) equipped with a 20x U Plan S Apochromat objective (NA 0.75 and working distance of $0.6 \mathrm{~mm}$ ) with a motorized magnification changer set to $1 \times$ and a camera (Orca ER-AG c4742-80-12AG; Hamamatsu Photonics). Images of the fluorescent ECM and Lifeact-GFP-expressing cells (acquired every $30 \mathrm{~min}$ for $27 \mathrm{~h}$ ) were first flat field corrected. Lifeact-GFP cells were identified by thresholding and connected component labeling. Fluorescent ECM images were photobleach corrected. Only the regions not underneath the cells were used to determine the appropriate photobleach corrections to ensure that ECM degradation was not mistaken for photobleaching. Cells were tracked through the entire experiment, and the change in ECM fluorescence underneath each cell was measured between each image. An empirical threshold for between image fluorescence change was selected to identify cells that degraded the matrix.

\section{Single-cell tracking}

Glass-bottomed culture dishes were coated with fibronectin $37^{\circ} \mathrm{C}$ for $1 \mathrm{~h}$. Coated dishes were then blocked with $5 \% \mathrm{BSA}$ at $37^{\circ} \mathrm{C}$ for $1 \mathrm{~h}$ to prevent nonspecific cell adhesion. Cells were plated on fibronectin-coated dishes for $8-12 \mathrm{~h}$ before imaging. Time-lapse microscopy was performed on an incubator fluorescent microscope (VivaView $\mathrm{FL}$ ) at $37^{\circ} \mathrm{C}$ and $5 \% \mathrm{CO}_{2}$ using a $20 \times$ objective with a motorized magnification changer set to $0.5 \times$. Cell speed was measured with ImageJ using the Manual Tracking plugin (National Institutes of Health).

\section{Wound-healing assay}

Glass-bottomed culture dishes were coated with $10 \mu \mathrm{g} / \mathrm{ml}$ fibronectin and thoroughly washed. $10^{6}$ cells were plated for $8-12 \mathrm{~h}$ before generating a scratch wound with a sterile P200 pipette tip. Images were acquired every $10 \mathrm{~min}$ for $12 \mathrm{~h}$ on an incubator microscope (VivaView $\mathrm{FL}$ ) at $37^{\circ} \mathrm{C}$ and $5 \% \mathrm{CO}_{2}$ using a $20 \times$ objective with a motorized magnification changer set to $0.5 x$. Wound edge detection at every time point was performed with ImageJ using methods as described by K. Straatman (Advanced Imaging Facilities, University of Leicester, Leicester, UK).

In brief, wound edges were first detected using the ImageJ function Process $\rightarrow$ Find Edges, sharpened, and thresholded. The Find Edges function was used again, and the lookup table for the resulting image was inverted. Next, the Analyze Particles function was applied with a lower bound set to 10,000 (background). Relative wound closure was quantified from measurements at every time point relative to the initial time point.

\section{Immunofluorescent cell staining}

For immunofluorescent staining, the cells were fixed, stained, and mounted as described previously (Bear et al., 2002). Cells were fixed with $4 \%$ 
paraformaldehyde and permeabilized in $0.1 \%$ Triton $X-100$ in PBS for $10 \mathrm{~min}$ at room temperature. Cells were then blocked for $30 \mathrm{~min}$ in PBS containing $5 \%$ normal goat serum and $5 \%$ fatty acid-free BSA. Primary antibodies were applied to cells in PBS containing $1 \%$ BSA overnight at $4{ }^{\circ} \mathrm{C}$. After washing the cells three times in PBS, fluorescent dye-conjugated secondary antibodies were diluted to $1: 500$ in 1\% BSA in PBS and applied to the coverslips for $1 \mathrm{~h}$. After three washes in PBS, the coverslips were mounted onto slides with Fluoromount-G (Electron Microscopy Sciences). Images were captured using a scanning confocal inverted microscope (FluoView FV 1000; Olympus) equipped with a 40 or $100 \times$ U Plan Fluor $\mathrm{N}$ objective (NA 1.30). Maximum intensity projections were generated using FluoView software (Olympus).

\section{Hanging droplet spheroids and spheroid collagen invasion}

Hanging droplet spheroids were generated according to modified methods of those previously described (Timmins and Nielsen, 2007). Cells were trypsinized and resuspended in media at $0.5-1 \times 10^{5}$ cells $/ \mathrm{ml} .20 \mu \mathrm{l}$ cell suspension was placed into each well of a 60-well minitray (Nunc; Thermo Fisher Scientific). The minitray was inverted and placed into a local humidification chamber. The humidification chamber was prepared by placing a 60-mm tissue culture dish (with lid removed) filled with $8 \mathrm{ml}$ PBS inside a $150-\mathrm{mm}$ tissue culture dish. Cells were incubated at $37^{\circ} \mathrm{C} 5 \% \mathrm{CO}_{2}$ for $4 \mathrm{~d}$ to allow spheroid formation at the medium-air interface. For collagen invasion assays, spheroids were embedded into collagen in 24-well tissue culture plates and followed for invasion for $3 \mathrm{~d}$. Collagen type I, high concentration $(8-11 \mathrm{mg} / \mathrm{ml})$ was purchased from BD. For confocal microscopy of spheroid invasion, cells were simultaneously fixed/permeabilized in $4 \%$ paraformaldehyde and $0.1 \%$ Triton X-100 for $1 \mathrm{~h}$. Cells were stained with fluorescent dye-conjugated phalloidin and Hoechst 33342 before visualizing on a microscope (FV1000) with a C Plan Fluor N objective (NA 0.3 and working distance of $9 \mathrm{~mm}$; 10XRC; Olympus).

\section{RNA preparation, RNA-Seq, and quantitative RT-PCR (qRT-PCR)}

Total RNA was isolated from cells with an RNeasy Plus Mini kit (QIAGEN) and validated to have RNA integrity numbers $>8.5$ using a 2100 Bioanalyzer (Agilent Technologies). For RNA-Seq, poly(A)+ RNA was enriched using the purification kit (Oligotex mRNA Mini Kit; QIAGEN) and fragmented with RNA fragmentation reagents (Ambion; Life Technologies). cDNA was synthesized with reverse transcriptase (SuperScript II; Life Technologies) by random priming followed by second-strand synthesis with DNA Polymerase I (Enzymatics) and purified using a PCR purification kit (QIAquick; QIAGEN). Libraries were prepared according to the manufacturer's specifications (Illumina). Sequencing was performed using 50-bp single-end reads (HiSeq 2000; Illumina). Reads were aligned to the reference mouse genome (mm9) using TopHat (Trapnell et al., 2009), and gene expression was estimated by calculating reads per kilobase per million reads (Mortazavi et al., 2008) for all Ensembl genes, analyzing only exonic reads. For GRT-PCR, cDNA was generated from total RNA (SuperScript II) using a 50:50\% mixture of random primers (Invitrogen) and the Oligo(dT) primer (Promega). This cDNA was used directly with TaqMan Gene Expression Master Mix and TaqMan Gene Expression Assays (Applied Biosystems) to be read on a qRT-PCR machine (ViiA 7; Applied Biosystems). Sik1 and Gapdh TaqMan probes were obtained from Life Technologies. QRT-PCR data were analyzed by the Pfaffl method (Pfaffl, 2001).

\section{Microfluidic device preparation}

Transparency masks were printed using a high-resolution printer (Fineline Imaging). The pattern for the chamber was fabricated on 4-inch silicon wafers using a two-step photolithography process. The first step involved a 5- $\mu \mathrm{m}$-tall layer of SU-8 (25) purchased from Microposit, and the microcapillaries were transferred to the wafer. After developing the first layer, a second 100-jm-tall layer of SU-8 (100) was applied to the same wafer, and after alignment, the channels were transferred to the wafer. After developing and postbaking, the silicon wafer was exposed to silane overnight. Polymethylsiloxane (PDMS) was then poured on the wafer and cured overnight at $70^{\circ} \mathrm{C}$. Individual PDMS devices were cut out from the wafer and placed in a clean dish until use. Ports were punched out in the devices. The devices were then washed with water and ethanol, blow dried, and plasma cleaned. The PDMS device was placed into contact with the glassbottomed dish immediately, ensuring that an irreversible seal was formed. The cell chamber ports were plugged with short pieces of tubing $(0.0025 \times$ 0.03125 inch; Upchurch Scientific).

\section{Chemotactic gradients}

The cell culture chamber was filled with $10 \mu \mathrm{g} / \mathrm{ml}$ fibronectin for $1 \mathrm{~h}$ at $37^{\circ} \mathrm{C}$ followed by flushing with sterile PBS. Cells were loaded into the cell culture chamber using a gel-loading pipette tip. The exit ports of the sink and source channels were connected to waste using tubing of an inner diameter of 0.015 inches. 100-pl glass syringes (model 1710 TLL $100 \mu$ SYR; Gastight; Hamilton) were connected to 27.5-gauge needles connected to the tubing. The source syringe and tubing were filled with serum-free DMEM containing the indicated chemoattractant and $10 \mu \mathrm{g} / \mathrm{ml}$ TRITC-dextran to visualize the gradient. The sink syringe and tubing were filled with serum-free DMEM. The tubing was then inserted into the source and sink channels, respectively, and the syringe pump was operated at a flow rate of $20 \mathrm{nl} / \mathrm{min}$. A stable gradient was then established in the cell culture chamber within 30 min and typically remained stable for $18 \mathrm{~h}$ as monitored by TRITC-dextran fluorescent intensity.

\section{Haptotaxis gradients}

Identical chambers as in the chemotaxis experiments were prepared. After bonding device to dish, gradients were formed by diffusion via the addition of $400 \mu \mathrm{g} / \mathrm{ml}$ Cy5-fibronectin to the source channel followed by washing with sterile PBS. The gradient was visualized by epifluorescence imaging and performing a line scan before seeding cells in the culture chamber. Additionally, a before and after wash-in protocol was developed in which the PDMS devices were plasma cleaned for $1 \mathrm{~min}$ and not dried at $70^{\circ} \mathrm{C}$ to allow removal of the PDMS after forming the fibronectin gradient. Cells were observed during haptotaxis for a minimum of $7 \mathrm{~h}$, at which time the drug was added and the same cells were observed for an additional $8-12 \mathrm{~h}$. This allowed matched drug versus nondrug treatment to confirm that any effects of the drugs on haptotaxis are not caused by chamber-to-chamber variability. For 3D haptotaxis assays, cells were first seeded in $1 \mathrm{mg} / \mathrm{ml}$ acid-neutralized collagen, after which the fibronectin gradient was formed.

\section{Directional migration image acquisition and analysis}

Chemotaxis and 3D haptotaxis assays were performed on a microscope (Disk Scanning Unit; Olympus) with a 20x objective using MetaMorph imaging software (Molecular Devices). Images were collected every $10 \mathrm{~min}$ for $>12 \mathrm{~h}$. Haptotaxis assays on 2D were performed on a microscope (VivaView FL) using a $20 x$ objective with a motorized magnification changer set to $0.5 x$ and at the same time interval. Individual cells were manually tracked using ImageJ software Manual Tracking plugin. Cells in 3D were tracked in $x-y$ as in 2D assays. The tracks obtained were further analyzed using the chemotaxis tool developed by ibidi (Chemotaxis and Migration Tool). This analysis tool was used to extract the FMI, persistence, and velocity of cell tracks from the manual tracking results. Data were analyzed using Prism (GraphPad Software) and displayed as mean $\pm 95 \%$ confidence intervals. Statistical analysis for these parameters was subsequently performed using a twotailed $t$ test.

\section{Online supplemental material}

Fig. S1 depicts the strategy for reconstitution of LKB I-deficient melanoma cell lines. Fig. S2 shows that $L K B 1$ is required for haptotaxis in multiple cell lines. Fig. S3 summarizes the results of evaluating the role of LKB 1 substrates in haptotaxis. Fig. S4 shows that MARK family kinases are required for haptotaxis. Fig. S5 reveals that a DN form of MARK enhances melanoma invasion into 3D collagen. Video 1 shows a live-cell invadopodia assay of WM-266-4 cells expressing Lifeact-GFP. Video 2 shows a LKB1-null melanoma cell migration in a wound-healing assay. Video 3 shows LKBI addback melanoma cell migration in a wound-healing assay. Table S1 lists the results from RNA-Seq analysis of LKB1-null and addback melanoma cells and is provided online as an Excel (Microsoft) file. Online supplemental material is available at http://www.jcb .org/cgi/content/full/jcb.201404067/DC 1 .

We thank Robert Currin of the University of North Carolina Olympus Imaging Research Center for assistance with imaging and members of the Bear laboratory for critical discussions. We also thank the University of North Carolina Flow Cytometry Facility for sorting of fluorescence cell lines.

This work was supported by National Institutes of Health grants to N.E. Sharpless (CA 163896), I.J. Davis (CA166447), and J.E. Bear (GM083035) and a University Cancer Research Fund innovation award to J.E. Bear and S.M. Gomez. J.E. Bear is also supported by the Howard Hughes Medical Institute.

The authors declare no competing financial interests.

Submitted: 11 April 2014

Accepted: 26 September 2014 


\section{References}

Artym, V.V., Y. Zhang, F. Seillier-Moiseiwitsch, K.M. Yamada, and S.C Mueller. 2006. Dynamic interactions of cortactin and membrane type matrix metalloproteinase at invadopodia: defining the stages of invadopodia formation and function. Cancer Res. 66:3034-3043. http://dx.doi .org/10.1158/0008-5472.CAN-05-2177

Asada, N., K. Sanada, and Y. Fukada. 2007. LKB1 regulates neuronal migration and neuronal differentiation in the developing neocortex through centrosomal positioning. J. Neurosci. 27:11769-11775. http://dx.doi.org/ 10.1523/JNEUROSCI.1938-07.2007

Baas, A.F., J. Kuipers, N.N. van der Wel, E. Batlle, H.K. Koerten, P.J. Peters, and H.C. Clevers. 2004. Complete polarization of single intestinal epithelial cells upon activation of LKB1 by STRAD. Cell. 116:457-466. http://dx.doi.org/10.1016/S0092-8674(04)00114-X

Banerjee,S., S.J.Buhrlage,H.T.Huang,X.Deng,W.Zhou,J.Wang, R. Traynor,A.R Prescott, D.R. Alessi, and N.S. Gray. 2014. Characterization of WZ4003 and HTH-01-015 as selective inhibitors of the LKB1-tumour-suppressoractivated NUAK kinases. Biochem. J. 457:215-225. http://dx.doi.org/10 1042/BJ20131152

Bardeesy, N., M. Sinha, A.F. Hezel, S. Signoretti, N.A. Hathaway, N.E. Sharpless, M. Loda, D.R. Carrasco, and R.A. DePinho. 2002. Loss of the Lkb1 tumour suppressor provokes intestinal polyposis but resistance to transformation. Nature. 419:162-167. http://dx.doi.org/10.1038/nature01045

Barnes, A.P., B.N. Lilley, Y.A. Pan, L.J. Plummer, A.W. Powell, A.N. Raines, J.R. Sanes, and F. Polleux. 2007. LKB1 and SAD kinases define a pathway required for the polarization of cortical neurons. Cell. 129:549-563. http://dx.doi.org/10.1016/j.cell.2007.03.025

Bear, J.E., T.M. Svitkina, M. Krause, D.A. Schafer, J.J. Loureiro, G.A. Strasser, I.V. Maly, O.Y. Chaga, J.A. Cooper, G.G. Borisy, and F.B. Gertler. 2002. Antagonism between Ena/VASP proteins and actin filament capping regulates fibroblast motility. Cell. 109:509-521. http://dx.doi.org/10.1016/ S0092-8674(02)00731-6

Berginski, M.E., S.J. Creed, S. Cochran, D.W. Roadcap, J.E. Bear, and S.M Gomez. 2014. Automated analysis of invadopodia dynamics in live cells. PeerJ. 2:e462. http://dx.doi.org/10.7717/peerj.462

Bosenberg, M., V. Muthusamy, D.P. Curley, Z. Wang, C. Hobbs, B. Nelson, C. Nogueira, J.W. Horner II, R. Depinho, and L. Chin. 2006. Characterization of melanocyte-specific inducible Cre recombinase transgenic mice. Genesis. 44:262-267. http://dx.doi.org/10.1002/dvg.20205

Cai, L., T.W. Marshall, A.C. Uetrecht, D.A. Schafer, and J.E. Bear. 2007. Coronin 1B coordinates Arp2/3 complex and cofilin activities at the leading edge. Cell. 128:915-929. http://dx.doi.org/10.1016/j.cell.2007.01.031

Cai, L., A.M. Makhov, D.A. Schafer, and J.E. Bear. 2008. Coronin 1B antagonizes cortactin and remodels Arp2/3-containing actin branches in lamellipodia. Cell. 134:828-842. http://dx.doi.org/10.1016/j.cell.2008.06.054

Carretero, J., T. Shimamura, K. Rikova, A.L. Jackson, M.D. Wilkerson, C.L. Borgman, M.S. Buttarazzi, B.A. Sanofsky, K.L. McNamara, K.A Brandstetter, et al. 2010. Integrative genomic and proteomic analyses identify targets for Lkb1-deficient metastatic lung tumors. Cancer Cell. 17:547-559. http://dx.doi.org/10.1016/j.ccr.2010.04.026

Chaffer, C.L., and R.A. Weinberg. 2011. A perspective on cancer cell metastasis Science. 331:1559-1564. http://dx.doi.org/10.1126/science.1203543

Chapin, S.J., C.M. Lue, M.T. Yu, and J.C. Bulinski. 1995. Differential expression of alternatively spliced forms of MAP4: a repertoire of structurally different microtubule-binding domains. Biochemistry. 34:2289-2301. http://dx.doi.org/10.1021/bi00007a025

Chen, W.T. 1989. Proteolytic activity of specialized surface protrusions formed at rosette contact sites of transformed cells. J. Exp. Zool. 251:167-185. http://dx.doi.org/10.1002/jez.1402510206

Cheng, H., P. Liu, Z.C. Wang, L. Zou, S. Santiago, V. Garbitt, O.V. Gjoerup, J.D Iglehart, A. Miron, A.L. Richardson, et al. 2009. SIK1 couples LKB1 to p53-dependent anoikis and suppresses metastasis. Sci. Signal. 2:ra35. http://dx.doi.org/10.1126/scisignal.2000369

Chinnakkannu, P., V. Samanna, G. Cheng, Z. Ablonczy, C.F. Baicu, J.R. Bethard, D.R. Menick, D. Kuppuswamy, and G. Cooper IV. 2010. Site-specific microtubule-associated protein 4 dephosphorylation causes microtubule network densification in pressure overload cardiac hypertrophy. J. Biol. Chem. 285:21837-21848. http://dx.doi.org/10.1074/jbc.M110.120709

Collins, S.P., J.L. Reoma, D.M. Gamm, and M.D. Uhler. 2000. LKB1, a novel serine/threonine protein kinase and potential tumour suppressor, is phosphorylated by cAMP-dependent protein kinase (PKA) and prenylated in vivo. Biochem. J. 345:673-680. http://dx.doi.org/10.1042/0264-6021:3450673

Contreras, C.M., S. Gurumurthy, J.M. Haynie, L.J. Shirley, E.A. Akbay, S.N Wingo, J.O. Schorge, R.R. Broaddus, K.K. Wong, N. Bardeesy, and D.H. Castrillon. 2008. Loss of Lkb1 provokes highly invasive endometrial adenocarcinomas. Cancer Res. 68:759-766. http://dx.doi.org/ 10.1158/0008-5472.CAN-07-5014
Courchet, J., T.L. Lewis Jr., S. Lee, V. Courchet, D.Y. Liou, S. Aizawa, and F. Polleux. 2013. Terminal axon branching is regulated by the LKB1NUAK1 kinase pathway via presynaptic mitochondrial capture. Cell. 153:1510-1525. http://dx.doi.org/10.1016/j.cell.2013.05.021

DiMilla, P.A., J.A. Stone, J.A. Quinn, S.M. Albelda, and D.A. Lauffenburger. 1993. Maximal migration of human smooth muscle cells on fibronectin and type IV collagen occurs at an intermediate attachment strength. J. Cell Biol. 122:729-737. http://dx.doi.org/10.1083/jcb.122.3.729

Drewes, G., A. Ebneth, U. Preuss, E.M. Mandelkow, and E. Mandelkow. 1997. MARK, a novel family of protein kinases that phosphorylate microtubule-associated proteins and trigger microtubule disruption. Cell. 89:297-308. http://dx.doi.org/10.1016/S0092-8674(00)80208-1

Friedl, P., and S. Alexander. 2011. Cancer invasion and the microenvironment: plasticity and reciprocity. Cell. 147:992-1009. http://dx.doi.org/10.1016/ j.cell.2011.11.016

Goodman, S.L., G. Risse, and K. von der Mark. 1989. The E8 subfragment of laminin promotes locomotion of myoblasts over extracellular matrix. J. Cell Biol. 109:799-809. http://dx.doi.org/10.1083/jcb.109.2.799

Goodwin, J.M., R.U. Svensson, H.J. Lou, M.M. Winslow, B.E. Turk, and R.J. Shaw. 2014. An AMPK-independent signaling pathway downstream of the LKB1 tumor suppressor controls Snail1 and metastatic potential. Mol. Cell. 55:436-450. http://dx.doi.org/10.1016/j.molcel.2014.06.021

Guldberg, P., P. thor Straten, V. Ahrenkiel, T. Seremet, A.F. Kirkin, and J. Zeuthen. 1999. Somatic mutation of the Peutz-Jeghers syndrome gene, LKB1/ STK11, in malignant melanoma. Oncogene. 18:1777-1780. http://dx.doi .org/10.1038/sj.onc.1202486

Guo, S., and K.J. Kemphues. 1995. par-1, a gene required for establishing polarity in $C$. elegans embryos, encodes a putative $\mathrm{Ser} / \mathrm{Thr}$ kinase that is asymmetrically distributed. Cell. 81:611-620. http://dx.doi.org/10.1016/ 0092-8674(95)90082-9

Hanahan, D., and R.A. Weinberg. 2000. The hallmarks of cancer. Cell. 100:5770. http://dx.doi.org/10.1016/S0092-8674(00)81683-9

Hearle, N., V. Schumacher, F.H. Menko, S. Olschwang, L.A. Boardman, J.J. Gille, J.J. Keller, A.M. Westerman, R.J. Scott, W. Lim, et al. 2006. Frequency and spectrum of cancers in the Peutz-Jeghers syndrome. Clin. Cancer Res. 12:3209-3215. http://dx.doi.org/10.1158/1078-0432.CCR-06-0083

Hemminki, A., D. Markie, I. Tomlinson, E. Avizienyte, S. Roth, A. Loukola, G. Bignell, W. Warren, M. Aminoff, P. Höglund, et al. 1998. A serine/ threonine kinase gene defective in Peutz-Jeghers syndrome. Nature. 391:184-187. http://dx.doi.org/10.1038/34432

Hezel, A.F., S. Gurumurthy, Z. Granot, A. Swisa, G.C. Chu, G. Bailey, Y. Dor, N. Bardeesy, and R.A. Depinho. 2008. Pancreatic LKB1 deletion leads to acinar polarity defects and cystic neoplasms. Mol. Cell. Biol. 28:2414-2425. http://dx.doi.org/10.1128/MCB.01621-07

Hompland, T., A. Erikson, M. Lindgren, T. Lindmo, and C. de Lange Davies. 2008. Second-harmonic generation in collagen as a potential cancer diagnostic parameter. J. Biomed. Opt. 13:054050. http://dx.doi.org/10.1117/ 1.2983664

Houde, V.P., M.S. Ritorto, R. Gourlay, J. Varghese, P. Davies, N. Shpiro, K. Sakamoto, and D.R. Alessi. 2014. Investigation of LKB1 Ser431 phosphorylation and Cys433 farnesylation using mouse knockin analysis reveals an unexpected role of prenylation in regulating AMPK activity. Biochem. J. 458:41-56. http://dx.doi.org/10.1042/BJ20131324

Illenberger, S., G. Drewes, B. Trinczek, J. Biernat, H.E. Meyer, J.B. Olmsted, E.M. Mandelkow, and E. Mandelkow. 1996. Phosphorylation of microtubuleassociated proteins MAP2 and MAP4 by the protein kinase p110mark. Phosphorylation sites and regulation of microtubule dynamics. J. Biol. Chem. 271:10834-10843. http://dx.doi.org/10.1074/jbc.271.18.10834

Jackson, E.L., N. Willis, K. Mercer, R.T. Bronson, D. Crowley, R. Montoya, T. Jacks, and D.A. Tuveson. 2001. Analysis of lung tumor initiation and progression using conditional expression of oncogenic K-ras. Genes Dev. 15:3243-3248. http://dx.doi.org/10.1101/gad.943001

Jeghers, H., V.A. McKusick, and K.H. Katz. 1949. Generalized intestinal polyposis and melanin spots of the oral mucosa, lips and digits; a syndrome of diagnostic significance. N. Engl. J. Med. 241:1031-1036. http://dx.doi .org/10.1056/NEJM194912292412601

Ji, H., M.R. Ramsey, D.N. Hayes, C. Fan, K. McNamara, P. Kozlowski, C. Torrice, M.C. Wu, T. Shimamura, S.A. Perera, et al. 2007. LKB1 modulates lung cancer differentiation and metastasis. Nature. 448:807-810. http://dx.doi.org/10.1038/nature06030

Jonkers, J., R. Meuwissen, H. van der Gulden, H. Peterse, M. van der Valk, and A. Berns. 2001. Synergistic tumor suppressor activity of BRCA2 and p53 in a conditional mouse model for breast cancer. Nat. Genet. 29:418-425. http://dx.doi.org/10.1038/ng747

Kemphues, K.J., J.R. Priess, D.G. Morton, and N.S. Cheng. 1988. Identification of genes required for cytoplasmic localization in early C. elegans embryos. Cell. 52:311-320. http://dx.doi.org/10.1016/S0092-8674(88)80024-2 
Kline, E.R., S. Muller, L. Pan, M. Tighiouart, Z.G. Chen, and A.I. Marcus. 2011. Localization-specific LKB1 loss in head and neck squamous cell carcinoma metastasis. Head Neck. 33:1501-1512. http://dx.doi.org/10.1002/ hed. 21638

Lim, W., N. Hearle, B. Shah, V. Murday, S.V. Hodgson, A. Lucassen, D. Eccles, I. Talbot, K. Neale, A.G. Lim, et al. 2003. Further observations on LKB1/ STK11 status and cancer risk in Peutz-Jeghers syndrome. Br. J. Cancer. 89:308-313. http://dx.doi.org/10.1038/sj.bjc.6601030

Liu, S., Y. Miao, C. Fan, Y. Liu, J. Yu, Y. Zhang, S. Dai, and E. Wang. 2013. Clinicopathologic correlations of liver kinase B1, E-cadherin, and N-cadherin expression in non-small cell lung cancer. Appl. Immunohistochem. Mol. Morphol. 21:334-340. http://dx.doi.org/10.1097/PAI.0b013e31826b128b

Liu, W., K.B. Monahan, A.D. Pfefferle, T. Shimamura, J. Sorrentino, K.T. Chan, D.W. Roadcap, D.W. Ollila, N.E. Thomas, D.H. Castrillon, et al. 2012. LKB1/STK11 inactivation leads to expansion of a prometastatic tumor subpopulation in melanoma. Cancer Cell.21:751-764.http://dx.doi.org/10 .1016/j.ccr.2012.03.048

Lizcano, J.M., O. Göransson, R. Toth, M. Deak, N.A. Morrice, J. Boudeau, S.A. Hawley, L. Udd, T.P. Mäkelä, D.G. Hardie, and D.R. Alessi. 2004. LKB1 is a master kinase that activates 13 kinases of the AMPK subfamily, including MARK/PAR-1. EMBO J. 23:833-843. http://dx.doi.org/10 .1038/sj.emboj.7600110

Lo, B., G. Strasser, M. Sagolla, C.D. Austin, M. Junttila, and I. Mellman. 2012. Lkb1 regulates organogenesis and early oncogenesis along AMPK-dependent and -independent pathways. J. Cell Biol. 199:1117-1130. http://dx.doi .org/10.1083/jcb.201208080

Lucas, E.P., I. Khanal, P. Gaspar, G.C. Fletcher, C. Polesello, N. Tapon, and B.J. Thompson. 2013. The Hippo pathway polarizes the actin cytoskeleton during collective migration of Drosophila border cells. J. Cell Biol. 201:875-885. http://dx.doi.org/10.1083/jcb.201210073

Magdalena, J., T.H. Millard, and L.M. Machesky. 2003. Microtubule involvement in NIH 3T3 Golgi and MTOC polarity establishment. J. Cell Sci. 116:743-756. http://dx.doi.org/10.1242/jcs.00288

Marcus, A.I., and W. Zhou. 2010. LKB1 regulated pathways in lung cancer invasion and metastasis. J. Thorac. Oncol. 5:1883-1886. http://dx.doi.org/ 10.1097/JTO.0b013e3181fbc28a

Martin, S.G., and D. St Johnston. 2003. A role for Drosophila LKB1 in anteriorposterior axis formation and epithelial polarity. Nature. 421:379-384. http://dx.doi.org/10.1038/nature01296

Marx, A., C. Nugoor, S. Panneerselvam, and E. Mandelkow. 2010. Structure and function of polarity-inducing kinase family MARK/Par-1 within the branch of AMPK/Snf1-related kinases. FASEB J. 24:1637-1648. http:// dx.doi.org/10.1096/fj.09-148064

McCarthy, A., C.J. Lord, K. Savage, A. Grigoriadis, D.P. Smith, B. Weigelt, J.S. Reis-Filho, and A. Ashworth. 2009. Conditional deletion of the Lkb1 gene in the mouse mammary gland induces tumour formation. J. Pathol. 219:306-316. http://dx.doi.org/10.1002/path.2599

Mejia-Gervacio, S., K. Murray, T. Sapir, R. Belvindrah, O. Reiner, and P.M. Lledo. 2012. MARK2/Par-1 guides the directionality of neuroblasts migrating to the olfactory bulb. Mol. Cell. Neurosci. 49:97-103. http:// dx.doi.org/10.1016/j.mcn.2011.10.006

Miyoshi, H., A. Deguchi, M. Nakau, Y. Kojima, A. Mori, M. Oshima, M. Aoki, and M.M. Taketo. 2009. Hepatocellular carcinoma development induced by conditional beta-catenin activation in Lkb1+/- mice. Cancer Sci. 100:2046-2053. http://dx.doi.org/10.1111/j.1349-7006.2009.01284.x

Mohseni, M., J. Sun, A. Lau, S. Curtis, J. Goldsmith, V.L. Fox, C. Wei, M. Frazier, O. Samson, K.K. Wong, et al. 2014. A genetic screen identifies an LKB1-MARK signalling axis controlling the Hippo-YAP pathway. Nat. Cell Biol. 16:108-117. http://dx.doi.org/10.1038/ncb2884

Monahan, K.B., G.I. Rozenberg, J. Krishnamurthy, S.M. Johnson, W. Liu, M.K. Bradford, J. Horner, R.A. Depinho, and N.E. Sharpless. 2010. Somatic p16(INK4a) loss accelerates melanomagenesis. Oncogene. 29:5809_ 5817. http://dx.doi.org/10.1038/onc.2010.314

Monypenny, J., D. Zicha, C. Higashida, F. Oceguera-Yanez, S. Narumiya, and N. Watanabe. 2009. Cdc42 and Rac family GTPases regulate mode and speed but not direction of primary fibroblast migration during plateletderived growth factor-dependent chemotaxis. Mol. Cell. Biol. 29:27302747. http://dx.doi.org/10.1128/MCB.01285-08

Mortazavi, A., B.A. Williams, K. McCue, L. Schaeffer, and B. Wold. 2008. Mapping and quantifying mammalian transcriptomes by RNA-Seq. Nat. Methods. 5:621-628. http://dx.doi.org/10.1038/nmeth.1226

Nguyen, H.B., J.T. Babcock, C.D. Wells, and L.A. Quilliam. 2013. LKB1 tumor suppressor regulates AMP kinase/mTOR-independent cell growth and proliferation via the phosphorylation of Yap. Oncogene. 32:4100-4109. http://dx.doi.org/10.1038/onc.2012.431

Olschwang, S., C. Boisson, and G. Thomas. 2001. Peutz-Jeghers families unlinked to STK11/LKB1 gene mutations are highly predisposed to primitive biliary adenocarcinoma. J. Med. Genet. 38:356-360. http://dx.doi.org/10 $.1136 / j m g .38 .6 .356$

Ou, W., S. Ye, W. Yang, Y. Wang, Q. Ma, C. Yu, H. Shi, Z. Yuan, G. Zhong, J. Ren, et al. 2012. Enhanced antitumor effect of cisplatin in human NSCLC cells by tumor suppressor LKB1. Cancer Gene Ther. 19:489498. http://dx.doi.org/10.1038/cgt.2012.18

Partanen, J.I., T.A. Tervonen, M. Myllynen, E. Lind, M. Imai, P. Katajisto, G.J. Dijkgraaf, P.E. Kovanen, T.P. Mäkelä, Z. Werb, and J. Klefström. 2012. Tumor suppressor function of Liver kinase B1 (Lkb1) is linked to regulation of epithelial integrity. Proc. Natl. Acad. Sci. USA. 109:E388E397. http://dx.doi.org/10.1073/pnas.1120421109

Petrie, R.J., A.D. Doyle, and K.M. Yamada. 2009. Random versus directionally persistent cell migration. Nat. Rev. Mol. Cell Biol. 10:538-549. http:// dx.doi.org/10.1038/nrm 2729

Pfaffl, M.W. 2001. A new mathematical model for relative quantification in real-time RT-PCR. Nucleic Acids Res. 29:e45. http://dx.doi.org/10.1093/ nar/29.9.e45

Ridley, A.J., M.A. Schwartz, K. Burridge, R.A. Firtel, M.H. Ginsberg, G. Borisy, J.T. Parsons, and A.R. Horwitz. 2003. Cell migration: integrating signals from front to back. Science. 302:1704-1709. http://dx.doi.org/ 10.1126/science. 1092053

Rodríguez-Fraticelli, A.E., M. Auzan, M.A. Alonso, M. Bornens, and F. Martín-Belmonte. 2012. Cell confinement controls centrosome positioning and lumen initiation during epithelial morphogenesis. J. Cell Biol. 198:1011-1023. http://dx.doi.org/10.1083/jcb.201203075

Rowan, A., V. Bataille, R. MacKie, E. Healy, D. Bicknell, W. Bodmer, and I. Tomlinson. 1999. Somatic mutations in the Peutz-Jeghers (LKB1/STKII) gene in sporadic malignant melanomas. J. Invest. Dermatol. 112:509511. http://dx.doi.org/10.1046/j.1523-1747.1999.00551.x

Sanchez-Cespedes, M., P. Parrella, M. Esteller, S. Nomoto, B. Trink, J.M. Engles, W.H. Westra, J.G. Herman, and D. Sidransky. 2002. Inactivation of LKB1/STK11 is a common event in adenocarcinomas of the lung. Cancer Res. 62:3659-3662.

Sapir, T., S. Sapoznik, T. Levy, D. Finkelshtein, A. Shmueli, T. Timm, E.M Mandelkow, and O. Reiner. 2008. Accurate balance of the polarity kinase MARK2/Par-1 is required for proper cortical neuronal migration. J. Neurosci. 28:5710-5720. http://dx.doi.org/10.1523/JNEUROSCI.0911-08.2008

Sapkota, G.P., A. Kieloch, J.M. Lizcano, S. Lain, J.S. Arthur, M.R. Williams, N. Morrice, M. Deak, and D.R. Alessi. 2001. Phosphorylation of the protein kinase mutated in Peutz-Jeghers cancer syndrome, LKB1/STK11, at Ser431 by $\mathrm{p} 90$ (RSK) and cAMP-dependent protein kinase, but not its farnesylation at Cys(433), is essential for LKB1 to suppress cell growth. J. Biol. Chem. 276:19469-19482. http://dx.doi.org/10.1074/jbc.M009953200

Shamloo, A., N. Ma, M.M. Poo, L.L. Sohn, and S.C. Heilshorn. 2008. Endothelial cell polarization and chemotaxis in a microfluidic device. Lab Chip. 8:1292-1299. http://dx.doi.org/10.1039/b719788h

Shaw, R.J., N. Bardeesy, B.D. Manning, L. Lopez, M. Kosmatka, R.A. DePinho, and L.C. Cantley. 2004a. The LKB1 tumor suppressor negatively regulates mTOR signaling. Cancer Cell. 6:91-99. http://dx.doi.org/10.1016/ j.ccr.2004.06.007

Shaw, R.J., M. Kosmatka, N. Bardeesy, R.L. Hurley, L.A. Witters, R.A. DePinho, and L.C. Cantley. 2004b. The tumor suppressor LKB1 kinase directly activates AMP-activated kinase and regulates apoptosis in response to energy stress. Proc. Natl. Acad. Sci. USA. 101:3329-3335. http://dx.doi.org/ 10.1073/pnas.0308061100

Shelly, M., L. Cancedda, S. Heilshorn, G. Sumbre, and M.M. Poo. 2007. LKB1/ STRAD promotes axon initiation during neuronal polarization. Cell. 129:565-577. http://dx.doi.org/10.1016/j.cell.2007.04.012

Su, G.H., R.H. Hruban, R.K. Bansal, G.S. Bova, D.J. Tang, M.C. Shekher, A.M. Westerman, M.M. Entius, M. Goggins, C.J. Yeo, and S.E. Kern. 1999. Germline and somatic mutations of the STK11/LKB1 Peutz-Jeghers gene in pancreatic and biliary cancers. Am. J. Pathol. 154:1835-1840. http:// dx.doi.org/10.1016/S0002-9440(10)65440-5

Timm, T., J.P. von Kries, X. Li, H. Zempel, E. Mandelkow, and E.M. Mandelkow. 2011. Microtubule affinity regulating kinase activity in living neurons was examined by a genetically encoded fluorescence resonance energy transfer/fluorescence lifetime imaging-based biosensor: inhibitors with therapeutic potential. J. Biol. Chem. 286:41711-41722. http://dx.doi.org/10 .1074/jbc.M111.257865

Timmins, N.E., and L.K. Nielsen. 2007. Generation of multicellular tumor spheroids by the hanging-drop method. Methods Mol. Med. 140:141-151. http://dx.doi.org/10.1007/978-1-59745-443-8_8

Trapnell, C., L. Pachter, and S.L. Salzberg. 2009. TopHat: discovering splice junctions with RNA-Seq. Bioinformatics. 25:1105-1111. http://dx.doi.org/ 10.1093/bioinformatics/btp120

Watts, J.L., D.G. Morton, J. Bestman, and K.J. Kemphues. 2000. The C. elegans par-4 gene encodes a putative serine-threonine kinase required for establishing embryonic asymmetry. Development. 127:1467-1475. 
Wingo, S.N., T.D. Gallardo, E.A. Akbay, M.C. Liang, C.M. Contreras, T. Boren, T. Shimamura, D.S. Miller, N.E. Sharpless, N. Bardeesy, et al. 2009. Somatic LKB1 mutations promote cervical cancer progression. PLoS ONE. 4:e5137. http://dx.doi.org/10.1371/journal.pone.0005137

Wu, C., S.B. Asokan, M.E. Berginski, E.M. Haynes, N.E. Sharpless, J.D. Griffith, S.M. Gomez, and J.E. Bear. 2012. Arp2/3 is critical for lamellipodia and response to extracellular matrix cues but is dispensable for chemotaxis. Cell. 148:973-987. http://dx.doi.org/10.1016/j.cell.2011.12.034

Xu, J., F. Wang, A. Van Keymeulen, M. Rentel, and H.R. Bourne. 2005. Neutrophil microtubules suppress polarity and enhance directional migration. Proc. Natl. Acad. Sci. USA. 102:6884-6889. http://dx.doi.org/10 $.1073 /$ pnas.0502106102

Yamaguchi, H., M. Lorenz, S. Kempiak, C. Sarmiento, S. Coniglio, M. Symons, J. Segall, R. Eddy, H. Miki, T. Takenawa, and J. Condeelis. 2005. Molecular mechanisms of invadopodium formation: the role of the NWASP-Arp2/3 complex pathway and cofilin. J. Cell Biol. 168:441-452. http://dx.doi.org/10.1083/jcb.200407076

Yamahashi, Y., Y. Saito, N. Murata-Kamiya, and M. Hatakeyama. 2011. Polarityregulating kinase partitioning-defective $1 \mathrm{~b}$ (PAR1b) phosphorylates guanine nucleotide exchange factor $\mathrm{H} 1$ (GEF-H1) to regulate RhoAdependent actin cytoskeletal reorganization. J. Biol. Chem. 286:4457644584. http://dx.doi.org/10.1074/jbc.M111.267021

Zagórska, A., M. Deak, D.G. Campbell, S. Banerjee, M. Hirano, S. Aizawa A.R. Prescott, and D.R. Alessi. 2010. New roles for the LKB1-NUAK pathway in controlling myosin phosphatase complexes and cell adhesion. Sci. Signal. 3:ra25. http://dx.doi.org/10.1126/scisignal.2000616 